\title{
Phytochemicals and biological activities of Ligularia species
}

\author{
Jun-Li YANG, ${ }^{a}$ Rui WANG, ${ }^{\text {b }}$ and Yan-Ping SHI ${ }^{\mathrm{a}, \mathrm{b}, *}$ \\ ${ }^{a}$ State Key Laboratory of Applied Organic Chemistry, Lanzhou University, Lanzhou 730000, China \\ ${ }^{b}$ Key Laboratory of Chemistry of Northwestern Plant Resources and Key Laboratory for Natural Medicine of Gansu Prov- \\ ince, Lanzhou Institute of Chemical Physics, Chinese Academy of Sciences, Lanzhou 730000, China
}

Received 23 June 2011; Accepted 25 July 2011

(C) The Author(s) 2011. This article is published with open access at Springerlink.com

\begin{abstract}
Ligularia, an important genus of the Compositae family, has captured the interest of natural product chemists for years. Phytochemical investigations on the title genus have led to isolation of hundreds of secondary metabolites with various skeletons. Herein, we summarized the chemical constituents of this genus and their biological activities over the past few decades.
\end{abstract}

\section{Introduction}

The genus Ligularia has been taxonomically placed in the Compositae (tribe Senecioneae) with more than 27 species used as folk remedies ${ }^{1}$. The systematic and in-depth phytochemical investigations on Ligularia species have resulted in hundreds of secondary metabolites with various skeletons and interesting biological activities have been discovered from this genus. The application of some Ligularia species in traditional medicines has been in period. For example, L. sagitta possess efficacies of relieving phlegm and cough, invigorating circulation of blood, soothing pain, and particularly curing rheumatoid arthritis ${ }^{2}$. L. fischeri has been used as a folk medicine for the treatment of coughs, inflammations, jaundice, scarlet fever, rheumatoidal arthritis, and hepatic diseases ${ }^{3}$. L. veitchiana was reported for the treatment of influenza, cough, ulcer and pulmonary tuberculosis ${ }^{4}$. L. lapathifolia has been used to treat cough and inflammation $^{5}$. Furthermore, L. sibirica and L. hodgsoni are used as herbal remedies to treat bronchitis, cough, asthma, and phthisis $^{6}$.

Searching for bioactive molecules from nature source has always been our interest ${ }^{7-12}$. In the past years, some Ligularia species, such as L. virgaurea spp. oligocephala ${ }^{9}, L$. myriocephala $^{13}$, and $L$. fischeri ${ }^{14}$, have been investigated in our lab from the viewpoint of phytochemistry. The promising results stimulated our interest in Ligularia species as a source of substances with chemical and biological diversity. Here we review the state of the art in the phytochemical investigation and biological activity evaluation of Ligularia species in recent years (1990.1 2011.6).

\section{Chemical Constituents}

*To whom correspondence should be addressed. E-mail: shiyp@licp.cas.cn
1.1 Sesquiterpenoids: As the major chemical constituents, there are 289 sesquiterpenoids reviewed. These sesquiterpenoids comprise eremophilane-type (1-1 to 1-210), bisabolane-type (1-211 to 1-242), oplopane-type (1-243 to 1248), guaiane and pseudoguaiane types (1-249 to 1-253), eudesmane type (1-254 to 1-258), and other skeleton types (1259 to 1-267) as well as dimers (1-268 to 1-289). The names and corresponding plant sources of these sesquiterpenoids were listed in Table $1^{1-6,9,13-99}$.

1.1.1 Eremophilane Sesquiterpenoids: Of the 368 secondary metabolites reviewed in this paper, there are 210 eremophilane sesquiterpenoids (1-1 to 1-210). Consequently, the eremophilane sesquiterpenoid is the most common phytochemical type. Thus, the taxonomic significance of eremophilane sesquiterpenoids for the genus Ligularia needs further study in future. Most of these eremophilane sesquiterpenoids were obtained in the form of lactones, and they can be divided into five groups from the structural viewpoint: a) eremophilane-12,8-olides (1-1 to 1-77); b) eremophilane-12,8(14,6 $\alpha)$-diolides (1-78 to 1-99); c) eremophilane-14,6 $\alpha$-olides (1-100 to 1-104); d) furaneremophilane sesquiterpenoids (1-105 to 1-129); e) other eremophilane sesquiterpenoids (1-130 to 1-210).

1.1.1.1 Eremophilane-12,8-olides: Eremophilane-12,8olides (1-1 to 1-77) are the most popular eremophilane lactones. Compounds 1-1 to 1-36 are eremophilane-12,8 $\alpha$ olides, while compounds 1-37 to 1-57 are eremophilane- $12,8 \beta$ olides. Of the structures 1-1 to 1-77, $\mathrm{H}_{\mathrm{a} / \mathrm{b}}-6, \mathrm{H}-8$, and $\mathrm{H}-10$ were always substituted by various substitutions, such as $\mathrm{OH}$, OAc, OAng, OMe, and OEt. In some cases (1-5, 1-8, 1-12, 113, 1-16 to 1-18, 1-22, 1-24 to 1-26, and 1-31 to 1-35), an epoxy group has been formed between $\mathrm{C}-1$ and $\mathrm{C}-10$. Furthermore, a double bond is often constructed between $\mathrm{C}-8$ and C-9 (1-58 to 1-73) or C-7 and C-8 (1-74 to 1-77). 
1.1.1.2 Eremophilane-12,8(14,6 $\alpha)$-diolides: Of such structures (1-78 to 1-99), an interesting phenomenon is that all H-6 protons are $\beta$-oriented. In addition, the $\mathrm{H}-8$ protons are often substituted by $\mathrm{OH}, \mathrm{OMe}$, or OEt, while a double bond is
99, the H-6 protons in structures 1-100 to 1-104 are all $\beta$ oriented. This phenomenon may show some relationships with the biosynthetic pathway of $14,6 \alpha$-olide moiety.

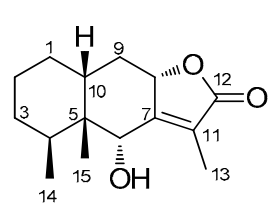

$1-1$<smiles>CC(=O)OC1=C(C)C(=O)OC2C[C@H]3CCCC(C)[C@]3(C)C12</smiles>

$1-2$<smiles>CCC(C)C(=O)OC1C2=C(C)C(=O)OC2CC2(O)CCC(O)C(C)C12C</smiles><smiles>CC1=C2O[C@@]3(O)C(C)CCC(O)C(O)C3(O)OC2(O)C(=O)O1</smiles>

$1-4$

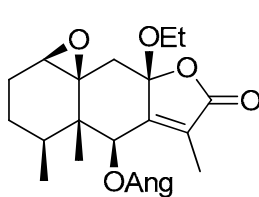

$1-5$

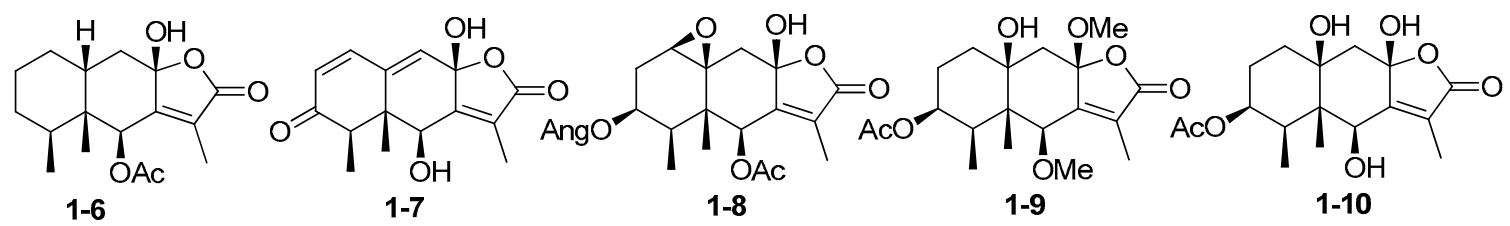

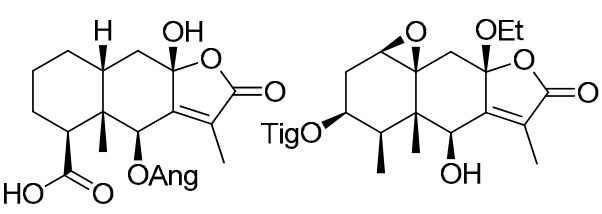

1-11<smiles>C=C(C)C(=O)OC1C2=C(C)C(=O)OC2(CC)C2(CC3OC32)C(C)CC1C</smiles>

1-13<smiles>CCC(C)OC1=C2C(=O)OC2(O)C[C@]2(O)CCC(OC(C)=O)C(C)C12C</smiles>

1-14

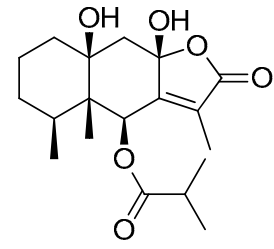

1-15<smiles>[R]C1C2=C(C)C(=O)OC2(O)CC23OC2CC(O)C(C)C13C</smiles>

$R$

1-16 OAC

1-17 $\mathrm{OH}$

1-18 H

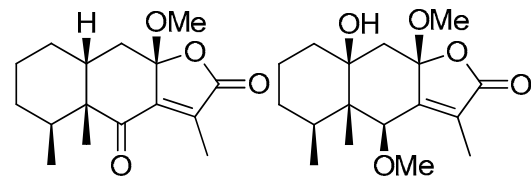

$1-19$<smiles>[CH]O[C@]12C[C@@]3(O)CCC[C@H](C)[C@]3(O)[C@@H](OC)C1=C(C)C(=O)O2</smiles>

1-21<smiles>CC1=C2[C@H](O)[C@@]3(C)[C@@H](C)CCC4O[C@]43C[C@]2(O)OC1=O</smiles>

1-22<smiles>CCC(C)C(=O)O[C@H]1C2=C(C)C(=O)OC2(OC)C[C@]2(O)CCC[C@H](C)[C@]12C</smiles>

1-23<smiles>C=C(CC(C)O)C(=O)O[C@H]1C2=C(C)C(=O)OC2(CC)[C@@]2(C)[C@H](C)CCC3OC312</smiles>

1-24<smiles>C=C(CO)C(=O)O[C@H]1C2=C(C)C(=O)O[C@@]2(O)CC23OC2CC[C@H](C)[C@]13C</smiles>

1-25<smiles>C=C(CO)C(=O)O[C@H]1C2=C(C)C(=O)OC2(CC)CC23OC2CC[C@H](C)[C@]13C</smiles>

$1-26$

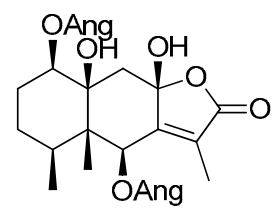

$1-27$ often constructed between C-8 and C-9 in some cases (1-91 to 1-94 and 1-99).

1.1.1.3 Eremophilane-14,6 $\alpha$-olides: As that of 1-78 to 1-
1.1.1.4 Furan-eremophilane Sesquiterpenoids: All of structures 1-105 to 1-129 possess a furan ring. Due to the sructural similarity, these compounds are put in one group in this review. Their most obvious structural characteristic is that

\section{照 Springer}


the C-6 positions always possess various substitutions, such as $\mathrm{OH}, \mathrm{OAc}$, and OAng. nature source. The structures 1-200 to 1-210 represent a rare carbon skeleton, and the probable biosynthetic pathway of

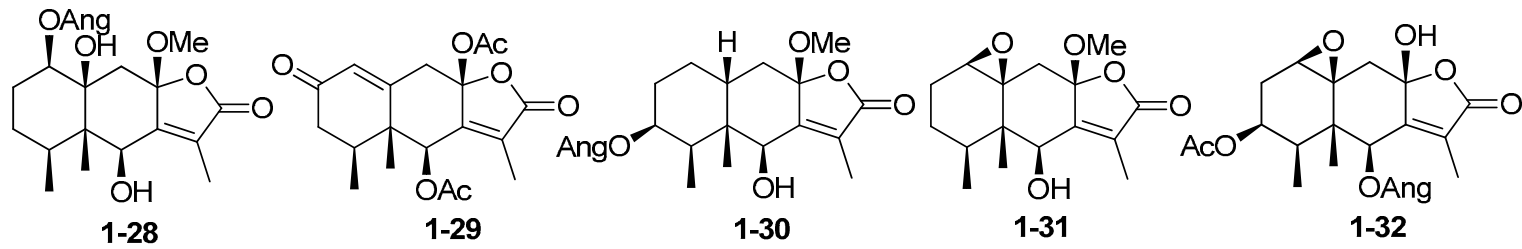<smiles>C=C(O)C(=O)O[C@H]1C2=C(C)C(=O)O[C@@]2(OC)C[C@@]23O[C@H]2CC[C@H](C)[C@]13C</smiles><smiles>C=C(C(=O)OC)C(=O)OC1C2=C(C)C(=O)OC2(O)C[C@]23OC2CCC(C)[C@]13C</smiles><smiles>C=C(C)C(=O)O[C@H]1C2=C(C)C(=O)O[C@@]2(O)C[C@]23O[C@H]2C[C@H](OC(C)=O)[C@@H](C)[C@@]13C</smiles>

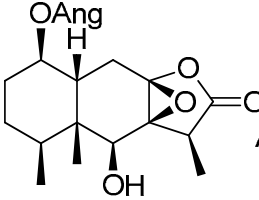<smiles>CO[C@H]1C2=C(C)C(=O)O[C@H]2C=C2CC[C@H](OC(C)=O)[C@@H](C)[C@]21C</smiles>

$1-36$

$1-37$<smiles>CCC(C)C(=O)O[C@H]1C2=C(C)C(=O)O[C@H]2C[C@]2(O)CC[C@H](OC(C)=O)[C@H](C)[C@]1(C)[C@H]2C</smiles>

1-39<smiles>COC(=O)[C@H]1CCC[C@H]2C[C@]3(O)OC(=O)C(C)=C3[C@@H](O)[C@]21C</smiles>

$1-40$<smiles>[R]C1C2=C(C)C(=O)O[C@@]2(OCC)C[C@H]2CCC[C@@H](C(=O)O)[C@@]12C</smiles>

$\mathrm{R}$

1-41 $\alpha$-OAng

$1-42 \beta$-OAng<smiles>CC(=O)O[C@H]1CC[C@@]2(O)C[C@]3(O)OC(=O)C(C)=C3[C@@H](O)[C@@]2(C)[C@H]1C</smiles>

$1-43$<smiles>CC1=C2C[C@]3(C)C(=C[C@]2(O)OC1=O)C(=O)CC[C@H]3C</smiles>
$1-44$<smiles>CCC(C)C(=O)O[C@H]1C2=C(C)C(=O)O[C@@]2(OC)C[C@@]2(O)CC[C@H](OC(C)C)[C@H](C)[C@]12C</smiles><smiles>C=C([As])C(=O)O[C@H]1C2=C(C)C(=O)O[C@@]2(OC)CC2CC[C@@H]3O[C@]3(C)[C@@]21C</smiles>

$1-50$<smiles>CCC(C)C(C)C(=O)O[C@H]1C2=C(C)C(=O)O[C@@]2(OC)C[C@]2(O)CCC[C@H](C)[C@]12C</smiles>

$1-51$<smiles>CO[C@H]1C2=C(C)C(=O)O[C@@]2(O[14CH3])C[C@@H]2CCC[C@@H](C(=O)O)[C@]21C</smiles><smiles>C=C(C)C(=O)O[C@H]1C2=C(C)C(=O)OC2(CC)CC2CC[C@H]3O[C@]3(C)[C@@]21C</smiles>

$1-46$

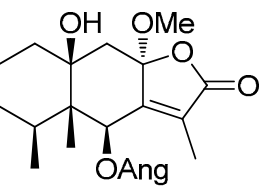<smiles>CC1=C2[C@H](O)[C@]3(C)C(=C[C@]2(O)OC1=O)C(=O)CC[C@H]3C</smiles>

$1-52$
1.1.1.5 Other Eremophilane Sesquiterpenoids: Besides above structures, there are still 81 eremophilane-type sesquiterpenoids (1-130-1-210) covered here. Compounds 1133 to 1-136 are isolated from the roots of $L$. fischeri in our $1 a b^{14,54}$, and 1-135 and 1-136 are obtained as a pair of epimers and their atructures have been confirmed by single-crystal Xray diffraction analysis ${ }^{14}$. Compound 1-137 is obtained as a sesquiterpenoid lactam $^{3}$, which is rarely discovered from such skeleton is proposed ${ }^{77,78}$.

1.1.2 Bisabolane Sesquiterpenoids: Bisabolane sesquiterpenoids 1-211 to 1-242 and their corresponding plant sources were indicated in Table 1 . Among them, $\mathrm{H}_{\mathrm{a} / \mathrm{b}}-1, \mathrm{H}-2$, $\mathrm{H}_{\mathrm{a} / \mathrm{b}}-8$, and $\mathrm{H}_{\mathrm{a} / \mathrm{b}}-10$ are always substituted by $\mathrm{OH}$ or OAng. Furthermore, there is often an epoxy group formed between $\mathrm{C}$ 3 and $C-4(1-213,1-214,1-218,1-219,1-227$ to $1-231$, and 1-

\section{是 Springer}


235) or between $\mathrm{C}-10$ and $\mathrm{C}-11$ (1-214, 1-215, 1-217 to 1-220, 1-223, 1-226 to 1-229, 1-233, and 1-235). In some cases (1237 to 1-242), ring $A$ is often oxygenated to benzonic moiety.
1.1.3 Oplopane Sesquiterpenoids: The six oplopane-type sesquiterpenoids 1-243 to 1-248, listed in Table 1, were all isolated from the roots of L. narynensis. Considering the structural characteristics, the C-3, C-4, C-8, and C-9 positions often possess various substitutions. Furthermore, in all of these

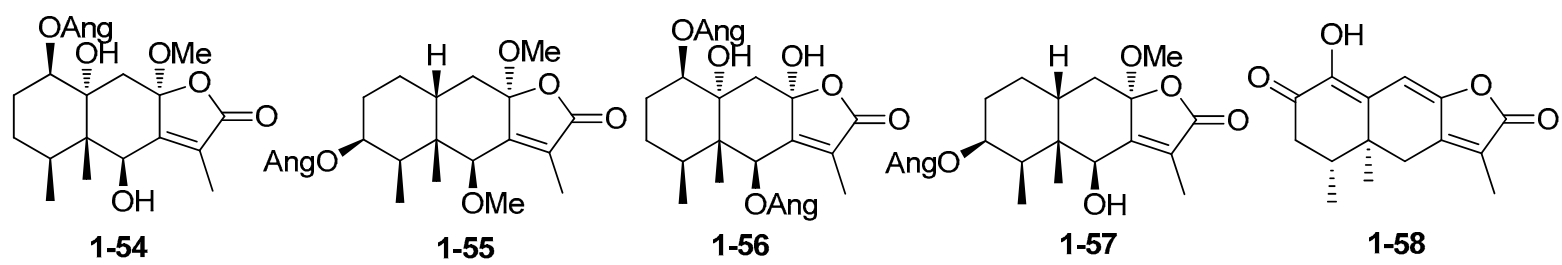

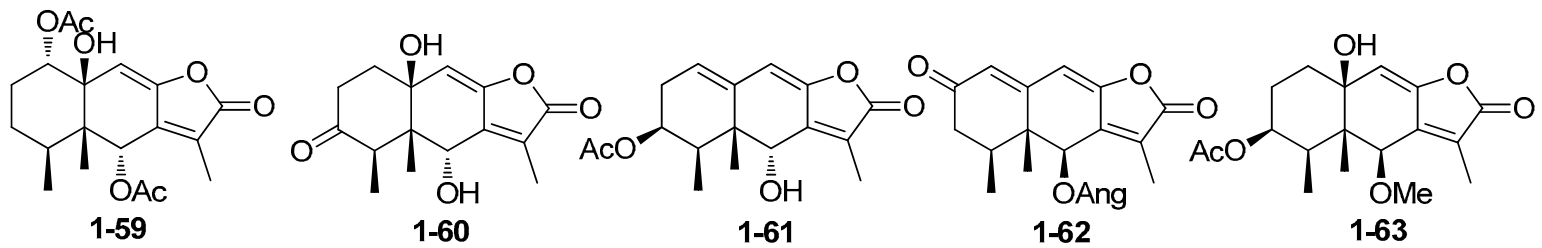

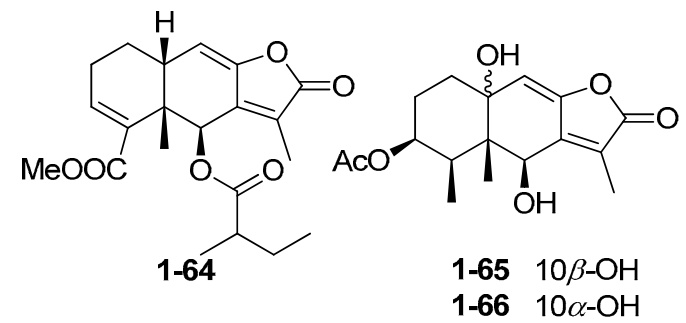<smiles>[R]C1C2=C(C)C(=O)OC2=C[C@]2(O)C(=O)CC[C@@H](C)[C@]12C</smiles>

$R$

$1-67 \alpha-\mathrm{OH}$

$1-68 \beta-\mathrm{OH}$ $1-69 \mathrm{H}$

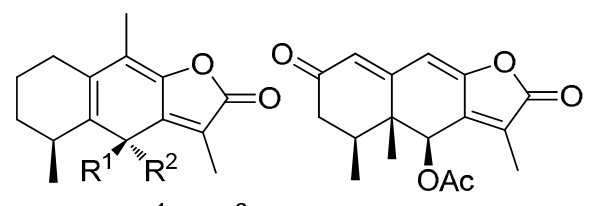

$R^{1} \quad R^{2}$

1-70 Me $\mathrm{H}$

1-71 $\mathrm{H} \quad \mathrm{Me}$
$1-72$

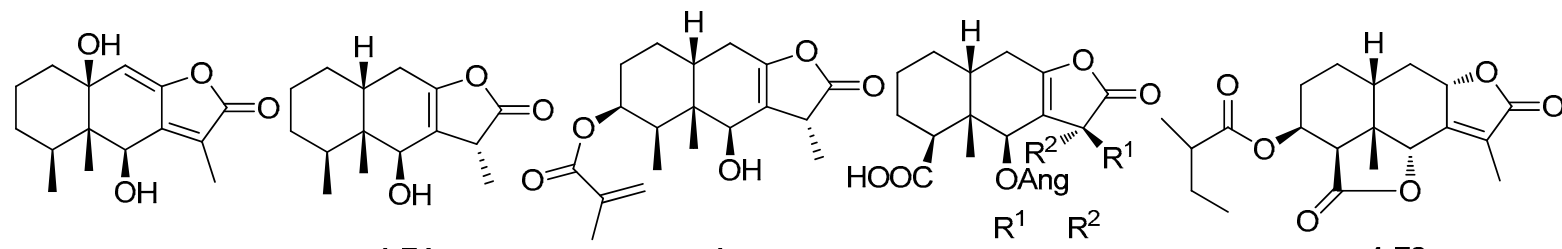

$1-73$

1-74

1-75

1.77 Me H

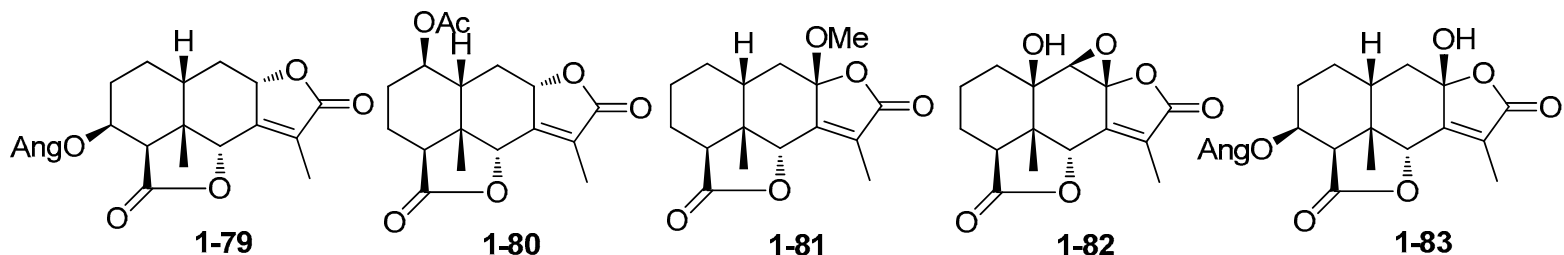

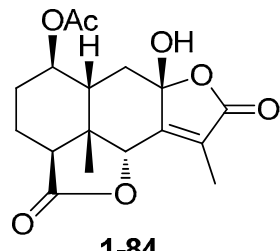

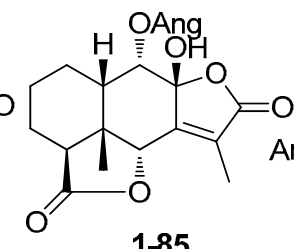

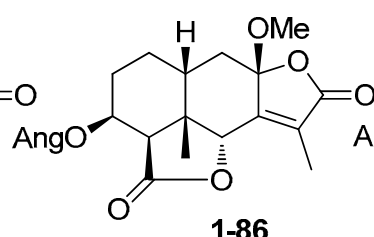

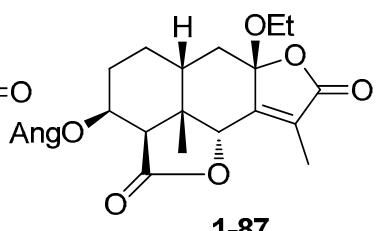

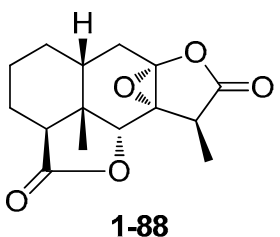


<smiles>CC1=C2[C@@H]3OC(=O)[C@@H]4CCCC(C[C@]2(O)OC1=O)[C@@]43C</smiles>

1-89<smiles>CC1=C2[C@H]3OC(=O)C3=CCC[C@@H]3C[C@H]2[C@@]3(C)C1=O</smiles>

$1-95$<smiles>CC1C(=O)OC2=C1[C@@H]1OC(=O)[C@@H]3CCC[C@H](C2)[C@@]31C</smiles>

$1-90$<smiles>[R]O[C@H]1C2=C(C)C(=O)OC2=C[C@@]2([R])CCC[C@@H]1C(=O)O2</smiles>

$R$

1-91 H $1-92 \mathrm{OH}$<smiles>CC1=C2C(=C[C@]3(O)CCC[C@@H]4C(=O)O[C@H]2[C@@]43C)OC1=O</smiles>

$1-93$<smiles>CCCCO[C@@H]1CC[C@@]2(O)C=C3OC(=O)C(C)=C3[C@@H]3OC(=O)[C@@H]1[C@@]32C</smiles>

$1-94$<smiles>[R]O[C@H]1C2=C(C)C(=O)O[C@@]2([R])C[C@H]2CCC=C3C(=O)O[C@]312</smiles>

$1-96 \mathrm{OH}$

1-97 OMe

1-98 OEt<smiles>CC1=C2C3=C[C@@H](CCC=C4C(=O)O[C@@H]42)[C@@]3(C)C1=O</smiles>

$1-99$<smiles>CC(=O)O[C@H]1CC[C@H]2C(=O)O[C@@H]3c4c(C)coc4C[C@]12C3C(C)=O</smiles>

$1-100$<smiles>CCCCCO[C@H]1CC[C@@H]2Cc3occ(C)c3[C@@H]3OC(=O)[C@@H]1[C@@]23C</smiles>

1-101

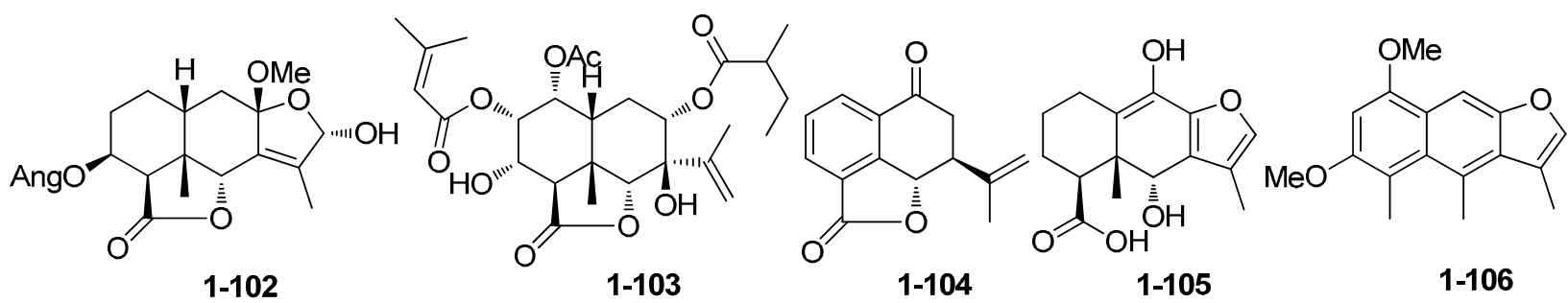<smiles>CO[C@H]1c2c(C)coc2CC2C=CC(=O)C(C)C21C</smiles>

1-107<smiles>CC(=O)O[C@H]1c2c(C)coc2C[C@@]2(O)CCC[C@H](C)[C@]12OC(C)=O</smiles>

1-108<smiles>[R7]Cc1coc2c1C([R2])[C@@]1(C)[C@@H](C)CC[C@H](O)[C@]1([R19])C2</smiles>

R1 R2 1-109 $\mathrm{OH} \quad \mathrm{H}$ $1-110 \mathrm{H} \quad \mathrm{OH}$ 1-111 $\mathrm{H} \quad \mathrm{H}$<smiles>[R20]C1c2c(C)coc2CC2=CC(=O)C[C@@H](C)[C@]21C</smiles>

$\mathrm{R}$ 1-112 Ang 1-113 Ac 1-114 H

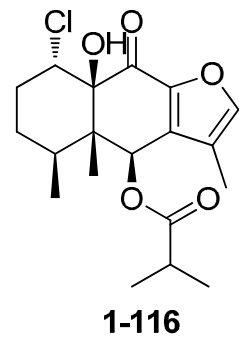<smiles>C=C(CO)C(=O)O[C@H]1c2c(C)coc2C[C@@]2(O)[C@@H](O)CC[C@H](C)[C@]12C</smiles>

1-115<smiles>C/C=C(/CO)C(=O)O[C@H]1c2c(C)coc2CC23OC2CC[C@H](C)[C@@]13C</smiles>

$1-117$<smiles>C/C(=C/CO)C(=O)O[C@H]1c2c(C)coc2CC2=CC(=O)C[C@H](C)[C@]21C</smiles>

$1-118$<smiles>Cc1coc2c1[C@H](O[14CH3])[C@]1(C)[C@@H](C)CCC(=O)[C@H]1C2</smiles>

$1-119$<smiles>Cc1coc2cc3c(c(C)c12)[C@@H](C)CC[C@H]3O</smiles>

$1-120$ structures, there is an epoxy group posited between $\mathrm{C}-11$ and C-12.

1.1.4 Other Sesquiterpenoids: Besides the above main sesquiterpenoids types, there were still guaiane-types 1-249 to
1-251, pseudoguaiane-types 1-252 and 1-253, and eudesmanetypes 1-254 to 1-258, as well as other types 1-259 to 1-267 being reviewed. Their names and corresponding plant sources were detailed in Table 1. 
<smiles>CCO[C@H]1c2occ(C)c2C[C@@]2(O)CC[C@H](OC(C)=O)[C@H](C)[C@@]12C</smiles>

1-121

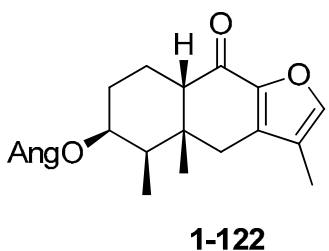

1-122<smiles>C=C(CO)C(=O)OC1c2c(C)coc2CC2=CCCC(C)[C@@]21C</smiles>

1-123<smiles>Cc1coc2c1C(OC(=O)C(C)C)[C@@]1(C)C(C)CCC(O)[C@@]1(O)C2=O</smiles>

1-124<smiles>CCC(C)C(=O)OC1c2c(C)coc2CC2(O)CCC(OC(C)=O)C(C)[C@]12C</smiles>

1-125<smiles>CC(=O)OC1CCC2(O)Cc3occ(C)c3[C@@](C)(C1OC(=O)C(C)C)[C@H]2C</smiles>

1-126<smiles>Cc1coc2c1[C@@H](O)[C@@]1(C)C(=CCCC1C)C2</smiles>

1-127<smiles>Cc1coc2cc3c(c(C)c12)C(C)C=CC3</smiles>

1-128<smiles>COc1cc(OC)c2cc3occc3c(C)c2c1C</smiles>

1-129<smiles>CC1C(=O)O[C@H]2[C@]1(O)C(=O)C[C@H]1CCC[C@H](C)[C@]12C</smiles>

1-130

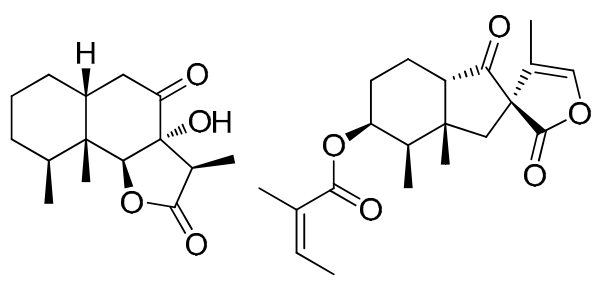

1-131

1-132<smiles>[R]C(C)C(=O)O[C@H]1C2=C(C)C(=O)OC2=C2C(O)CC[C@H](C)[C@]21C</smiles>

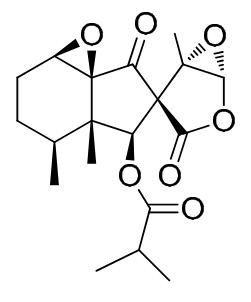

1-135

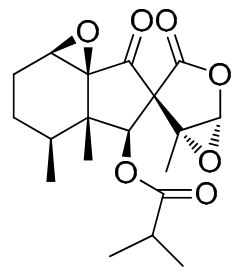

1-136<smiles>CC1=C2CC3(C)C(=CC2=CCCC3C)NC1=O</smiles><smiles>CC1=COC2C1(O)C(=O)C=C1C(=O)CCC(C)C12O</smiles>

1-142<smiles>C=C(C)[C@H]1CC2(C)C(=CC(=O)CC2C)C=C1OC(C)C</smiles>

1-143<smiles>C=C(CC)[C@@H]1C[C@]2(C)C(=CC1=O)C=C1CC[C@@H](OC(=O)CC)[C@@H](C(=C)C)C[C@@]1(C)C2C</smiles>

1-144

1-145

1-146

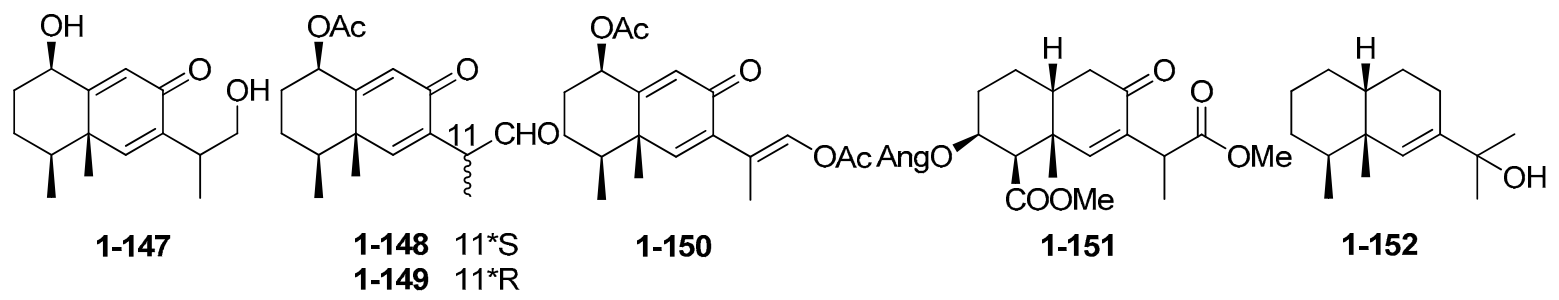

Compound 1-262, possessing a new carbon skeleton, was discovered from $L$. virgaurea spp. oligocephala ${ }^{9}$. The lactones 1-263 and 1-264 were obtained as a pair of isomers, and their structres were determined using extensive spectroscopic $\operatorname{methods}^{92}$. The novel structures 1-265 and 1-266 were obtained as sesquiterpenoid-coumarin dimers ${ }^{93}$, which are rarely discovered from nature source.

\section{照 Springer}




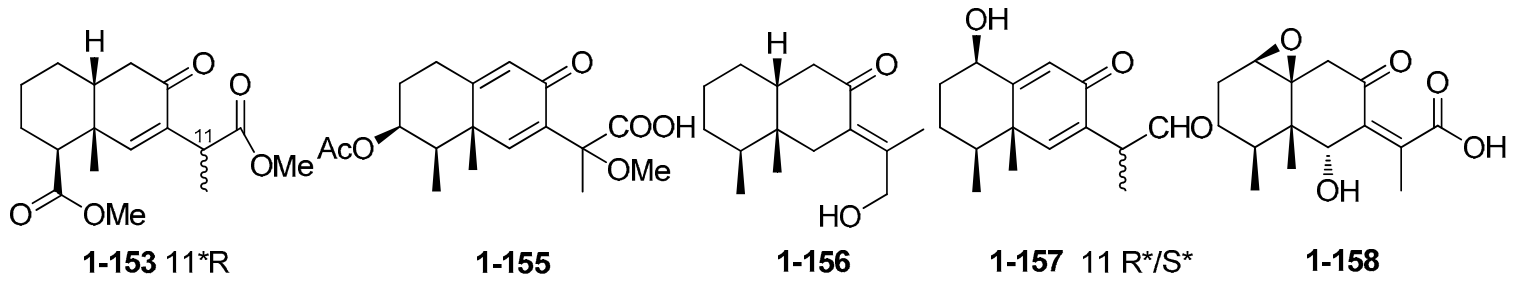

$1-15411^{*} S$<smiles>CCC(C)C(=O)OC(C)(C(=O)O)C1=CC2(C)C(=CC1=O)CCC(OC(C)=O)C2C</smiles>

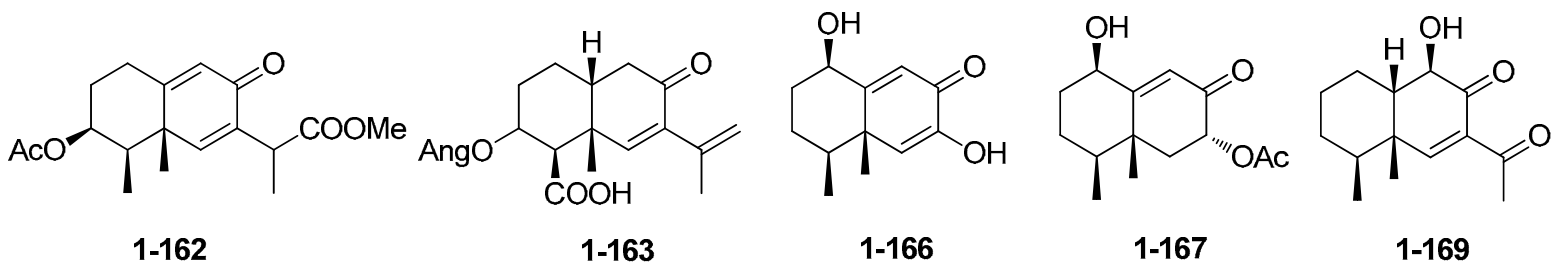

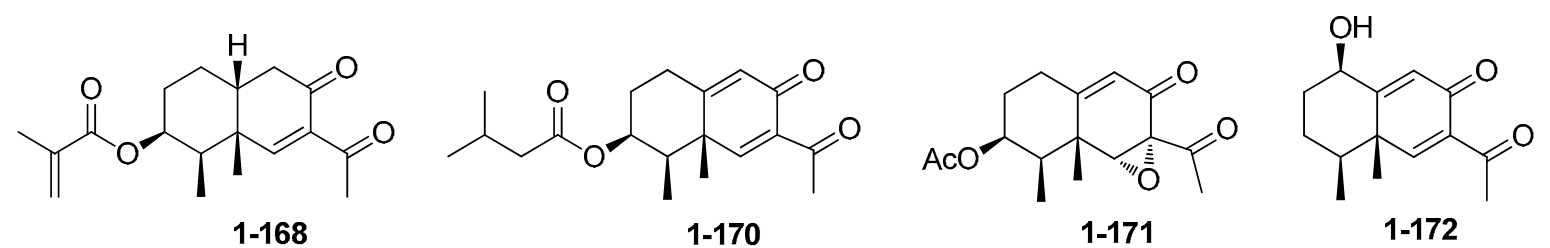<smiles>[R20]C(=O)c1cc(O)cc2c1C[C@H](C(=C)C)CC2</smiles><smiles>C=C(C)[C@H]1Cc2c(ccc(O)c2C)C[C@H]1O</smiles>

1-179<smiles>C=C(C)[C@H]1Cc2c(ccc(O)c2C)C(=O)[C@@H]1O</smiles>

1-180<smiles>C=C1CCC(O)c2ccc(C(C)=O)cc21</smiles>

1-181<smiles>CC(O)C1=CC2(C)C(C)CCC(O)C2C=CC1=O</smiles>

1-182

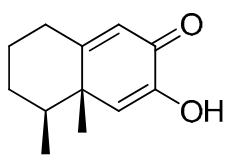

1-183<smiles>CC(=O)OC1CCC2=CC(=O)C(O)=CC2(C)C1C</smiles>

1-184<smiles>C=C(C)[C@H]1Cc2c(C=O)cccc2CC1O</smiles>

1-185<smiles>C=C(C)C1CCc2cc(O)cc(C(=O)OC)c2C1C</smiles>

1-186

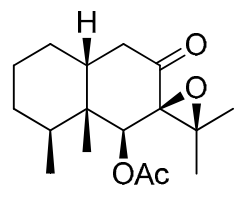

1-187 


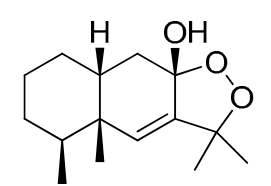

1-189<smiles>C[C@H]1CCC[C@H]2CC3=C(C(=O)O[C@H]3O)C(=O)[C@@]12C</smiles>

1-190<smiles>C=C(C)[C@@H]1CCc2ccc(O)c(C)c2C1</smiles>

1-191<smiles>COC(=O)[C@@]12O[C@H]1[C@@]1(C)C(C)CCC3OC31C2=O</smiles>

1-192<smiles>[CH]O[C@@H]1CC[C@@H]2CC(C(C)=O)=C[C@]2(O)[C@H]1C(C)=O</smiles>

1-193<smiles>COC(=O)C1=CCC[C@]2(C)CC(C(C)=O)=C[C@]12C</smiles><smiles>CC(=O)C1=C[C@]2(C)[C@@H](C)CCC[C@H]2C1</smiles>

1-195<smiles>COC(=O)[C@@H]1CCC[C@H]2CC(C(C)=O)=C[C@@]21C</smiles>

1-196<smiles>CC(=O)O[C@H]1CC[C@]2(O)CC(C(C)=O)=C[C@]2(C)[C@H]1C</smiles>
1-197<smiles>COC(=O)C1=C[C@]2(C)C(CC[C@@H](O[GaH2])[C@@H]2C(=O)OC)C1</smiles><smiles>C[C@H]1CC[C@@H](O)C2=CC(=O)C(C3(C)C=CC(O)CC(C(=O)O)=CO3)=C[C@]21C</smiles>

1-199

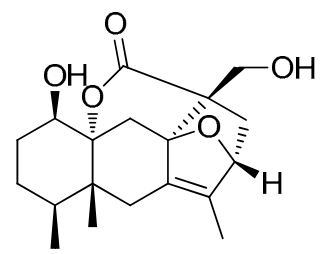

1-200

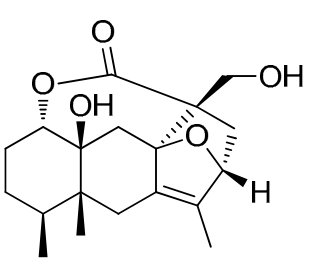

1-201

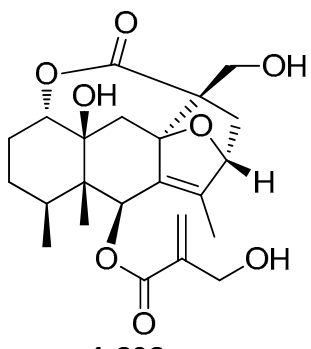

1-202

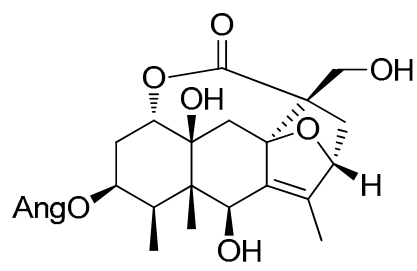

1-203

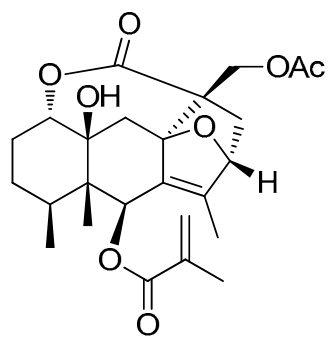

1-204

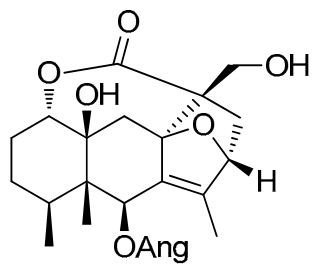

1-207

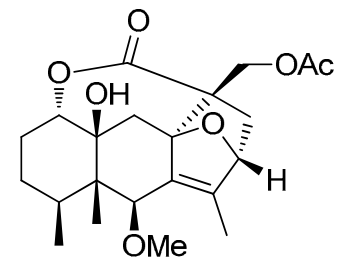

1-208
1-205

1-206
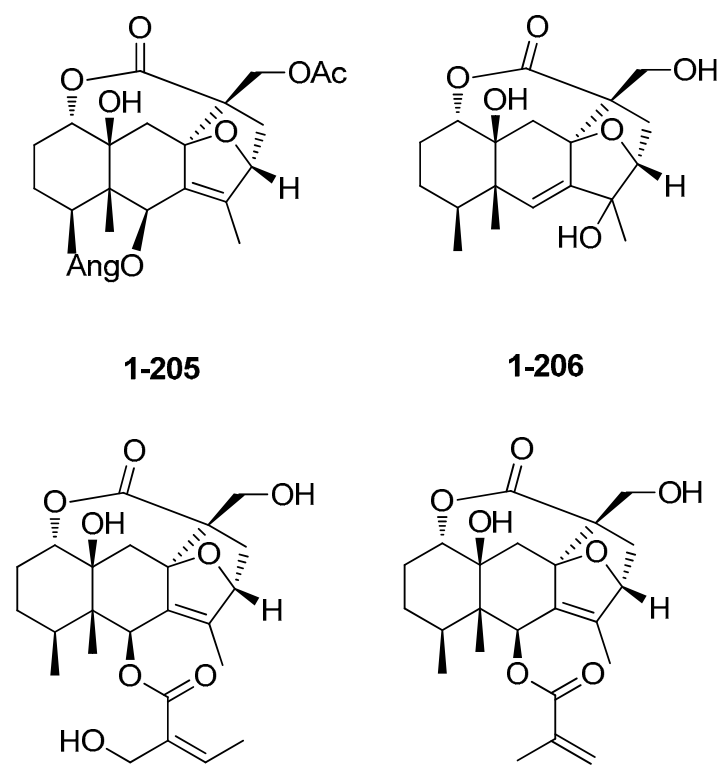

1-209

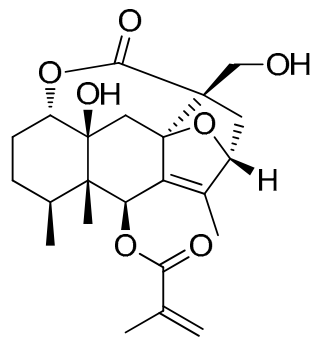

1-210

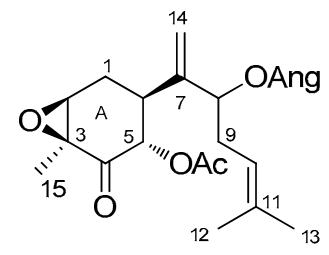

1-211<smiles>C=C(C(O)C(O)C(C)(C)O)C1CC(O)[C@](C)(O)[C@H](OCCCC)[C@H]1O</smiles>

1-212

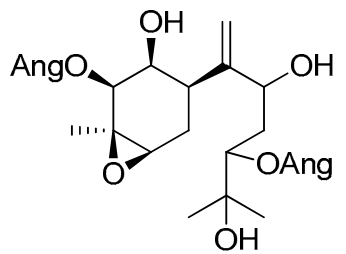

1-213<smiles>C=C(C(CC1OC1(C)C)O[Ga])[C@H]1C[C@H]2O[C@]2(C)[C@H](O[14CH3])[C@@H]1O</smiles>

$1-214$

望 Springer 


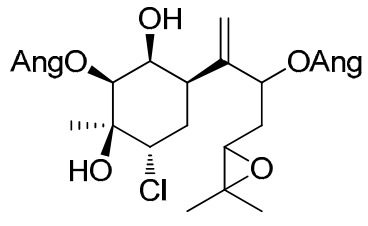

$1-215$

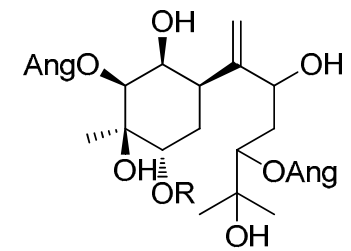<smiles>[R]CC1C[C@@H](C)[C@](C)(C(C)(C)C)OC1=O</smiles>

1-216

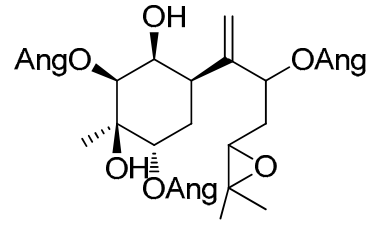

1-217

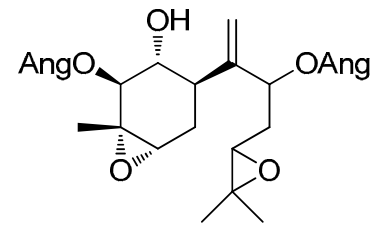

1-218

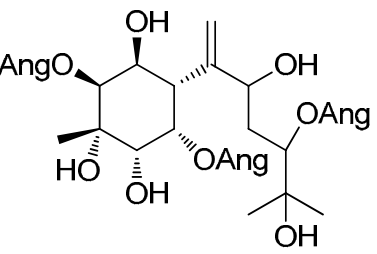
$1-222$<smiles>C=C(O[Ga])C(CC1OC1(C)C)[C@@H]1C[C@H](Cl)[C@](C)(O)[C@H](O)[C@H]1O[Na]</smiles>

1-226

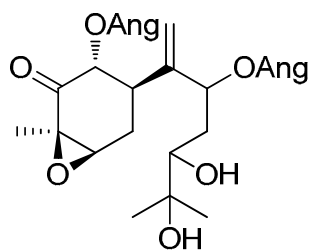

1-231<smiles>CCCCOC(CC1OC1(C)C)C(=O)[C@@H]1C[C@@H]2O[C@]2(C)[C@H](O)[C@@H]1OC(=O)C(C)CC</smiles>

1-235

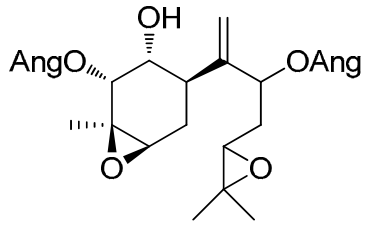

1-219

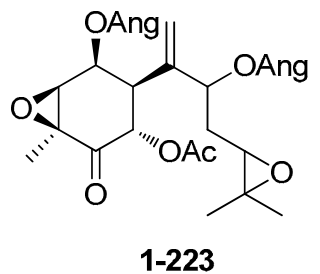

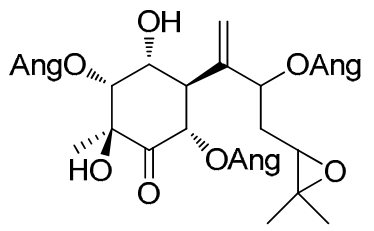

1-220

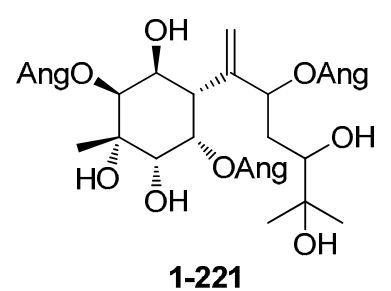

1-221

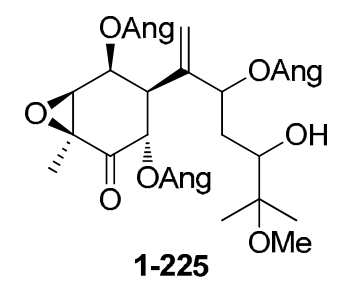

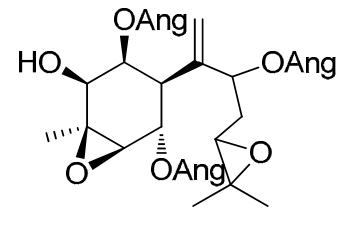

1-227<smiles>[R20]OC(CC1OC1(C)C)C(=C)[C@H]1C[C@H]2O[C@]2(C)[C@H]([R2])[C@@H]1O[Na]</smiles>

$\mathrm{R}$

1-228 $\beta$-OAng

1-229 $2 \mathrm{H}$

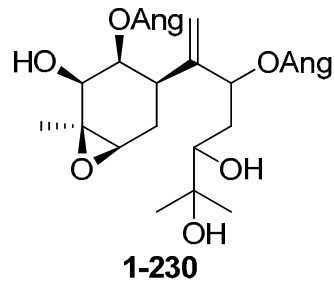

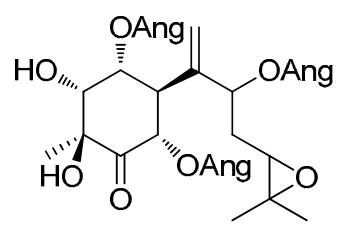

1-233

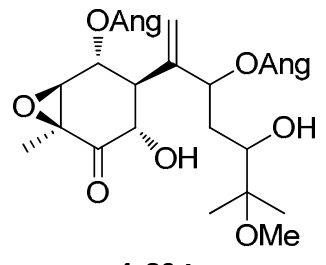

1-234<smiles></smiles>

1-238 


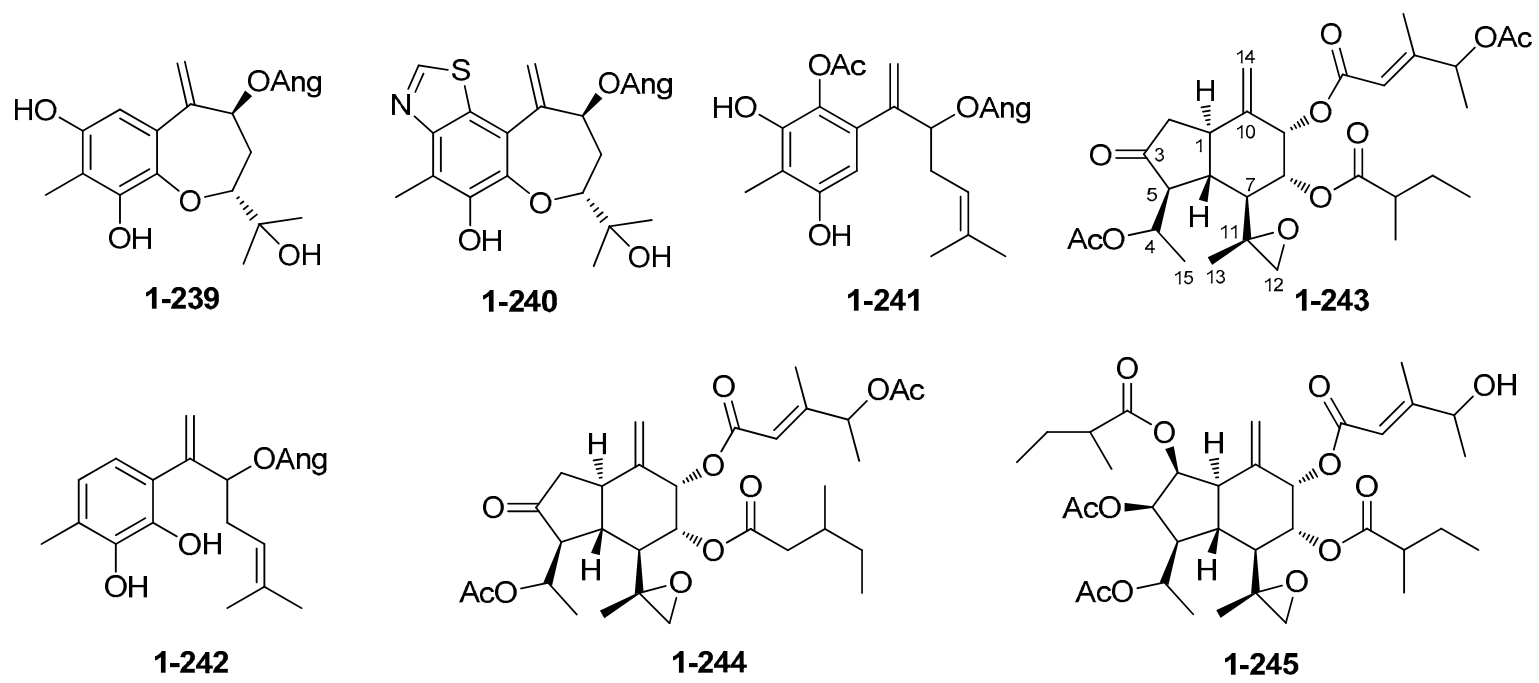

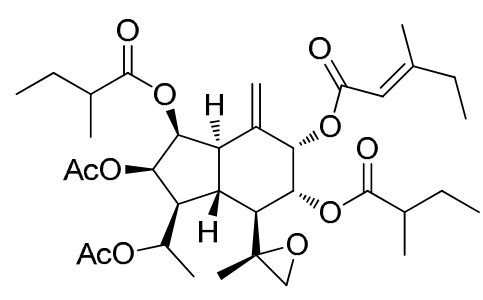

$1-246$

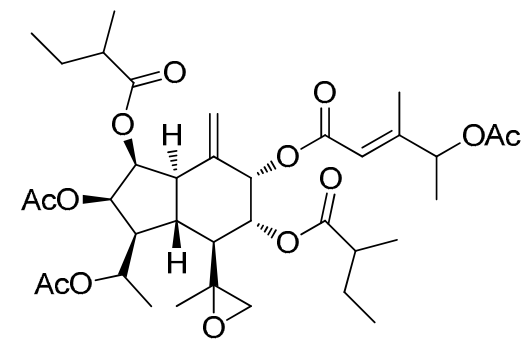

1-247

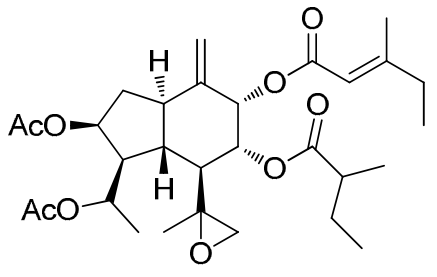

1-248

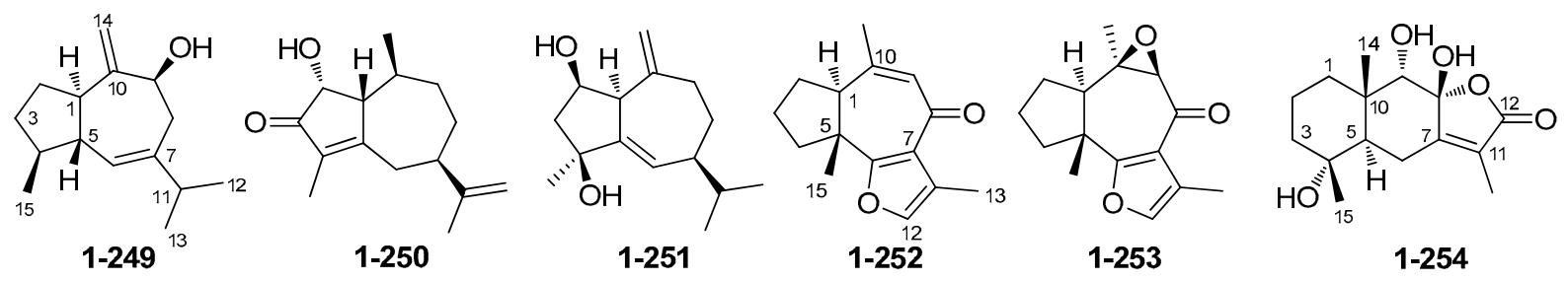

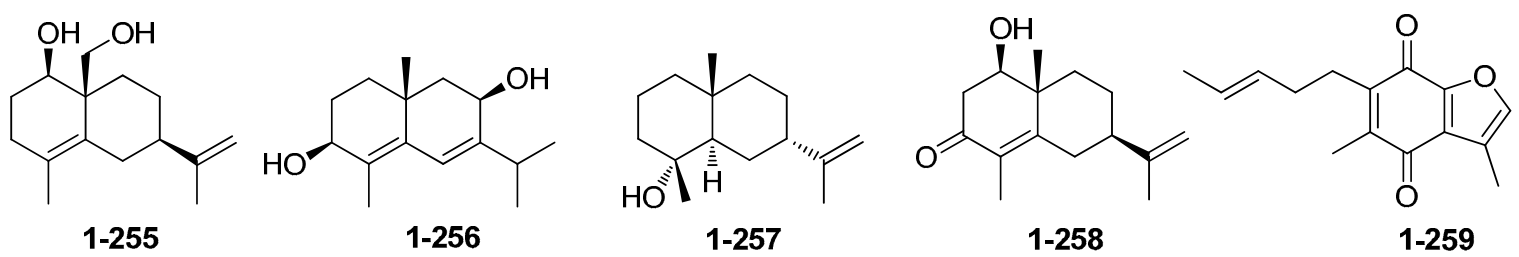<smiles>C/C=C/CCc1c(C)cc2c(C)coc2c1O</smiles>

$1-260 \stackrel{\mathrm{R}}{\mathrm{OH}}$

1-261 OMe<smiles></smiles>

1-262<smiles>CC1=C([C@]2(C)OC(=O)C3=C2[C@@H](C)CCC3)[C@H](O)OC1=O</smiles>

1-263<smiles>CC1=C(C)C(C)CCC1</smiles><smiles>CCC1(C)CC(=O)C(C)=C1C=CC(=O)O</smiles>

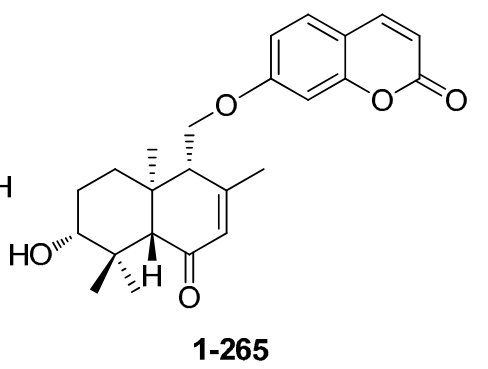



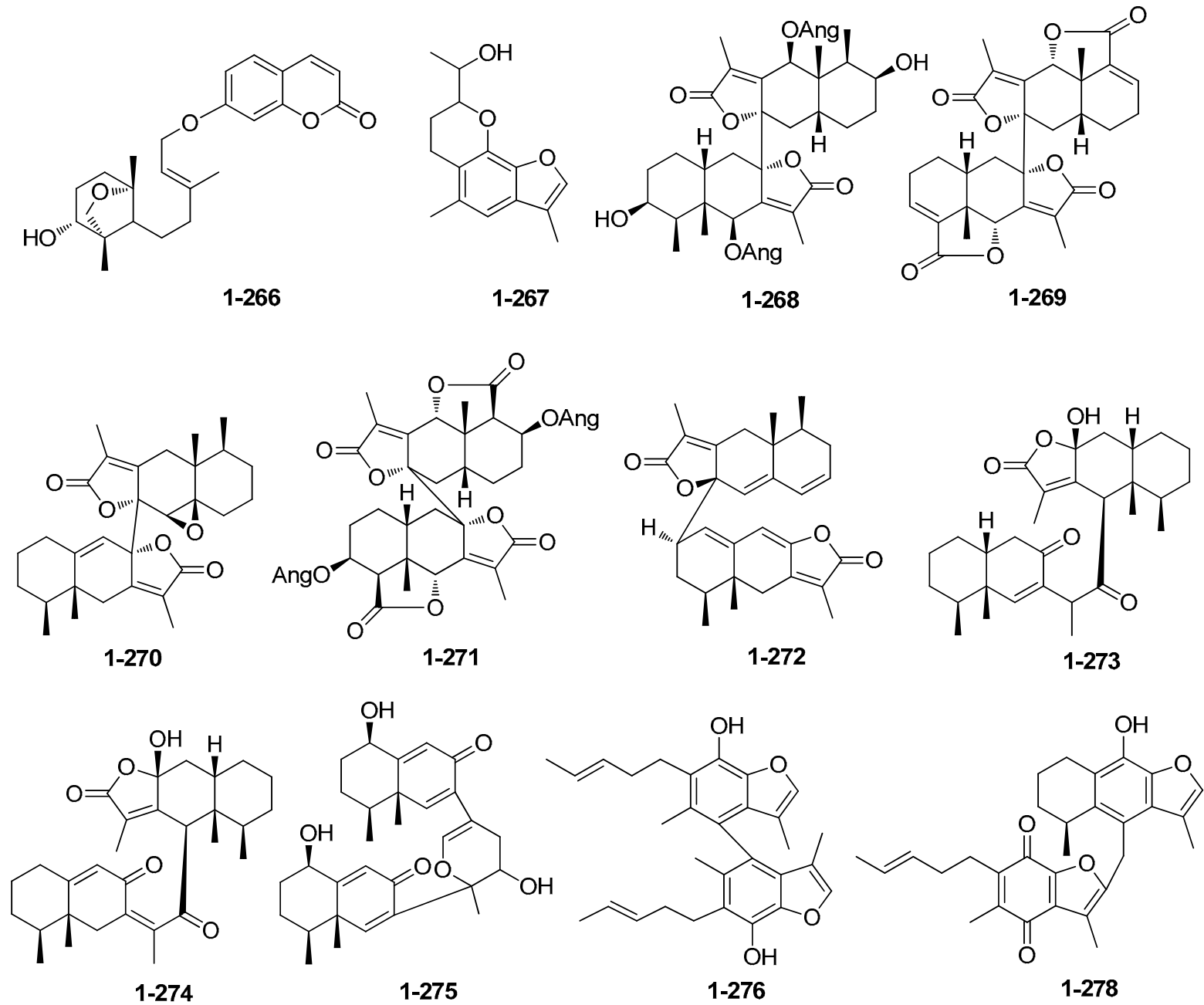<smiles>C/C=C/CCc1c(C)cc2c(c1O)OCC2(C)c1oc2c(C)c(CC/C=C/C)c(C)cc2c1O</smiles>

1-277

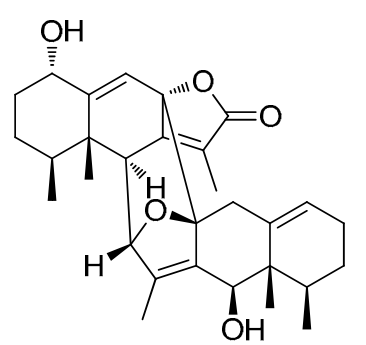

1-281

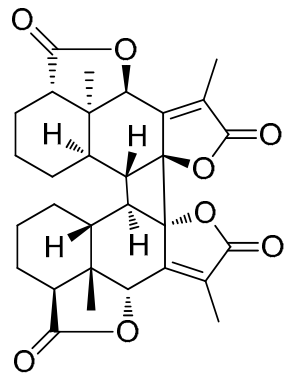

1-282
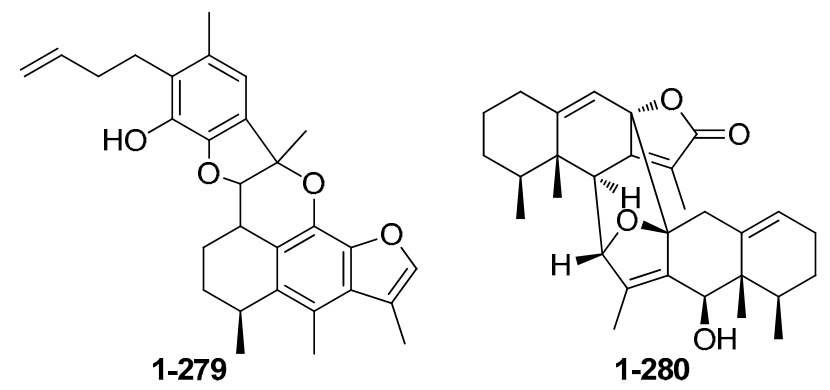

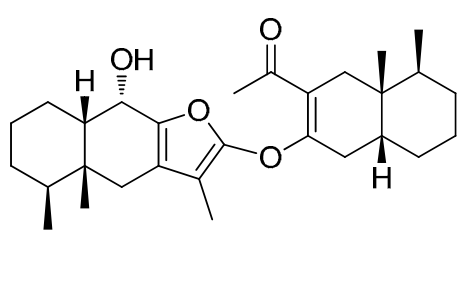

$1-283$

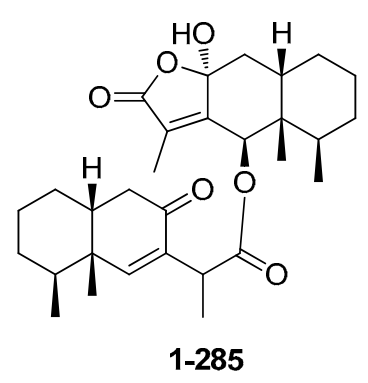


<smiles>Cc1c(OC2=C(C(=O)Oc3cc4c(c(C)c3C)C(C)CCC4)CC3CCCC(C)C23C)cc2c(c1C)CCCC2C</smiles>

1-284

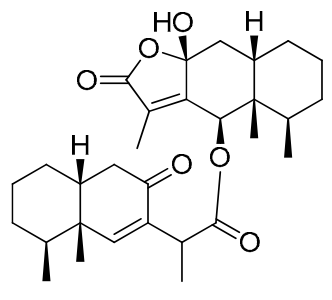

1-286

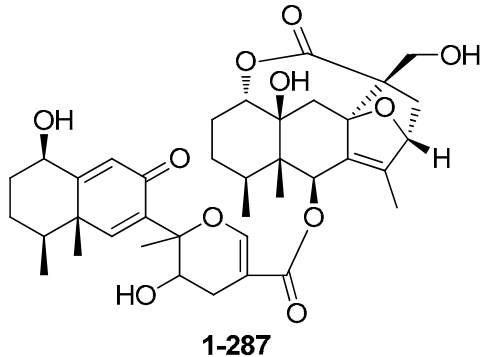

1-287<smiles>C/C(Oc1cc(C)c2c(c1)CCC=C2C(=O)O)=C(/C)Oc1cc(C)c2c(c1)CCC=C2C(=O)O</smiles>

$1-288$

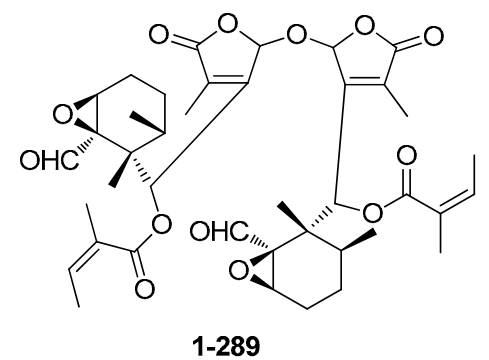

1.1.5 Sesquiterpenoid Dimers: The 22 sesquiterpenoid dimers 1-268 to 1-289 have been indicated in Table 1. Of these, structures 1-268 to 1-282 share the C-C linkage pattern, while $1-283$ to $\mathbf{1 - 2 8 9}$ share the $\mathrm{C}-\mathrm{O}-\mathrm{C}$ linkage pattern $^{1,3,31,38,44,53,57,63,77,95-99}$.

1.2 Monoterpenoids and Diterpenoids: The structures of monoterpenoids 2-1 and 2-2 and diterpenoids 2-3 to 2-5 were provided, and their names and plant sources were listed in Table $2^{15,62,88,100}$. Of them, structure $\mathbf{2 - 3}$ was isolated as a $\mathrm{C}_{19^{-}}$ diterpenoid carbon skeleton from L. sagitta, and its structure was further confirmed using single-crystal X-ray diffraction method $^{62}$.<smiles>CC(C)[C@H]1C(O)CC(C)(O)C(O)C1O</smiles>

2-1<smiles>CC(C)C1CC(O)C(C)(O)C(O)C1O</smiles>

2-2

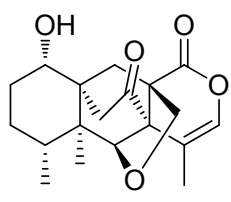

2-3<smiles>C=CC(=C)CCCC(C)CCCC(C)CCCC(C)C</smiles>

$2-4$<smiles>C=C/C(C)=C/C/C=C(\C)CC/C=C(\C)CCC=C(C)C</smiles>

2-5

1.3 Triterpenoids: The ten triterpenoids 3-1 to 3-10 mainly comprise oleane and norursane types. Their names and corresponding plant sources were indicated in Table $3^{40,47,100-}$ 105. Of them, compounds 3-3 and 3-4 were obtained as triterpenoid saponins from L. veitchiana ${ }^{102,103}$. Compounds 3-5 and 3-6 were isolated from L. intermedia in the form of 3,4- seco-oleanolic triterpene acids ${ }^{104}$. Compounds 3-7 and 3-8 are norursane-type triterpenoids and were isolated from $L$. tongolensis $^{47}$.

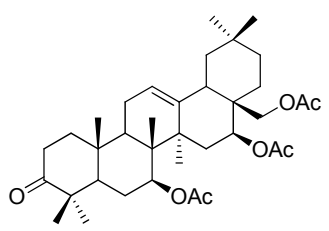

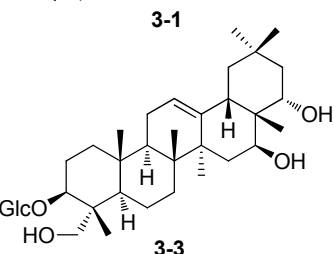

3-3

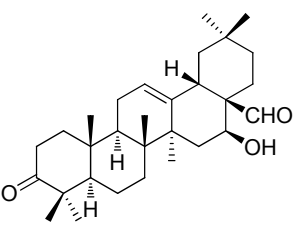

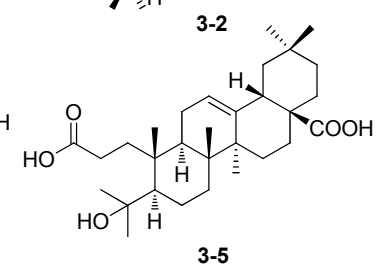<smiles>CC12CCCCC1CC=C1C3CCC(C)(C)C(C)(C)CCC3(C(=O)O)CCC12</smiles>

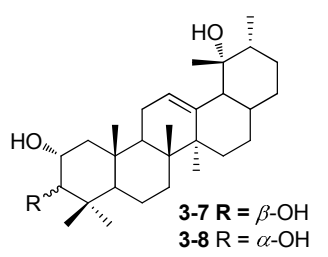

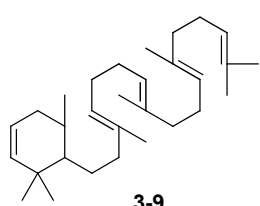

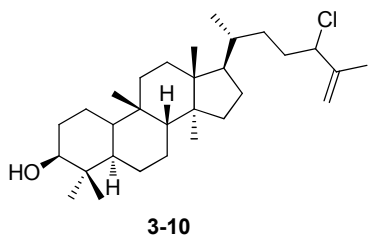

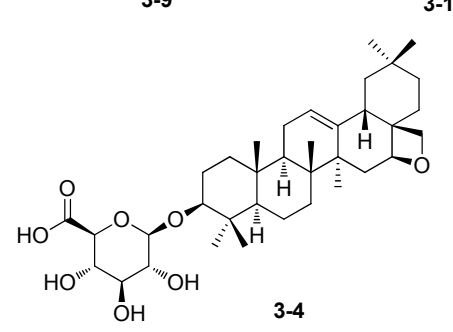

\section{是 Springer}


<smiles>CCC(CC(O)[C@@H](C)C1CC[C@H]2[C@H]3[C@H](O)C=C4C[C@@H](O)CC[C@]4(C)[C@H]3CC[C@]12C)C(C)C</smiles>

4-1

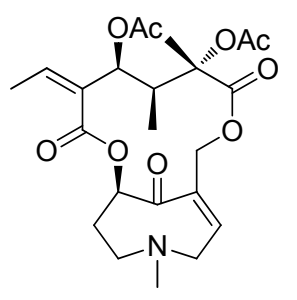

4-5

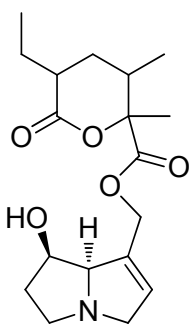

4-6

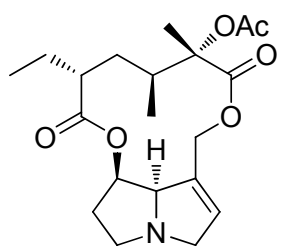

4-2

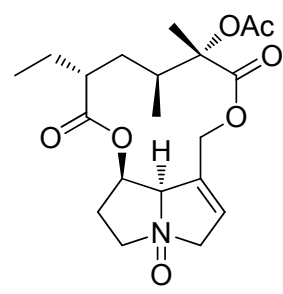

4-3<smiles>C=C/C(=C/[C@@H](C)[C@@](C)(OC(C)=O)C(=O)OCC1=CCN(C)CC[C@@H]1OC)C(=O)O</smiles>

4-4

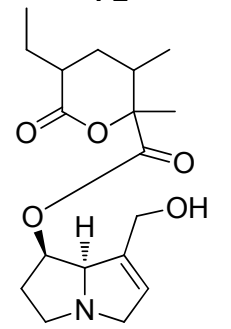

4-7<smiles></smiles>

4-8

4-9<smiles>Cc1cnncc1C(O)C(O)C(O)CO</smiles>

4-10<smiles>CC(C)N=c1cc2cc(O)c3cc(=NC(C)C)cc3cc(O)c2c1</smiles>

4-11<smiles>CCC(=N)N(C(=N)CC)C(=N)CC</smiles>

4-12<smiles>COc1cc(C(C)=O)cc2c(=O)c(C)c(C)oc12</smiles>

4-13<smiles>C=C(C)C1OC(=O)c2cc(C(C)=O)cc(OC)c2OC1=O</smiles><smiles>COc1cc(/C=C/CO)ccc1O</smiles><smiles>COc1cc2oc3c(C)ccc(C)c3c2cc1OC[14C](=O)[O-]</smiles><smiles>COc1cc2oc3c(C)ccc(C)c3c2cc1OC</smiles><smiles>COc1cc2oc3c(C)cc(C)c(C)c3c2cc1OC</smiles>

4-24<smiles>C=C(C)C(O)CC/C(C)=C/COc1ccc(/C=C/CO)cc1OC</smiles><smiles>COc1cc(/C=C/CO)ccc1OC/C=C(\C)C/C=C/C(C)(C)O</smiles>
4-26<smiles>C=C(C)C(O)CC/C(C)=C/COc1ccc(/C=C/CO)cc1OC</smiles> 
<smiles>COc1cc(/C=C/CO)ccc1OC/C=C(\C)C/C=C/C(C)(C)O</smiles>

4-28<smiles>COc1cc(/C=C/CO)cc(OC)c1OC/C=C(\C)C/C=C/C(C)(C)O</smiles><smiles>C=C(C)C(CC/C(C)=C/COc1c(OC)cc(/C=C/CO)cc1OC)OO</smiles>

4-32<smiles>CCCCCOC(=O)/C=C/c1ccc(O)c(OC)c1</smiles>

4-35<smiles>C=C(C)C1Cc2cc(C(C)=O)cc(O)c2O1</smiles>

4-39<smiles>COc1cc2oc(C(C)=O)cc2cc1CC=C(C)C</smiles><smiles>C=C(CC(C)(C)c1cc2cc(OC)c(OC)cc2o1)c1cc2cc(OC)c(OC)cc2o1</smiles><smiles>COc1cc2cc(/C=C(\C)C(C)(C)c3cc4cc(OC)c(OC)cc4o3)oc2cc1OC</smiles>
4-47

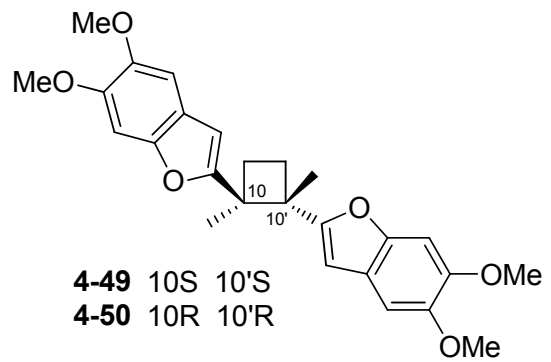<smiles>C=C(C)C(O)CC/C(C)=C/COc1c(OC)cc(/C=C/CO)cc1OC</smiles>

4-29<smiles>COc1cc(/C=C/CO)cc(OC)c1OC/C=C(\C)C/C=C/C(C)(C)O</smiles><smiles>[R]C=Cc1cc(OC)c(OC/C=C(\C)CCC=C(C)C)c(OC)c1</smiles>
4-33 $\mathrm{R}=\mathrm{CH}_{2} \mathrm{OH}$ 4-34 $\mathrm{R}=\mathrm{CHO}$<smiles>O=C(/C=C/c1ccc(OC(=O)/C=C/c2ccc(O)c(O)c2)cc1)O[O-]</smiles>

4-36

4-37<smiles>COc1cc(C(C)=O)cc2ccoc12</smiles>

4-40<smiles>[Z14]c1cc2c(cc1O)O/C(=C(/C)C(C)=O)C2=O</smiles><smiles>COc1cc2cc(C(C)(C)CC(C)(C)c3cc4cc(OC)c(OC)cc4o3)oc2cc1OC</smiles><smiles>C=C(C)C1Oc2cc(O)c(C(C)=O)cc2C1/C=C(/C)c1cc2cc(OC)c(OC)cc2o1</smiles><smiles></smiles>

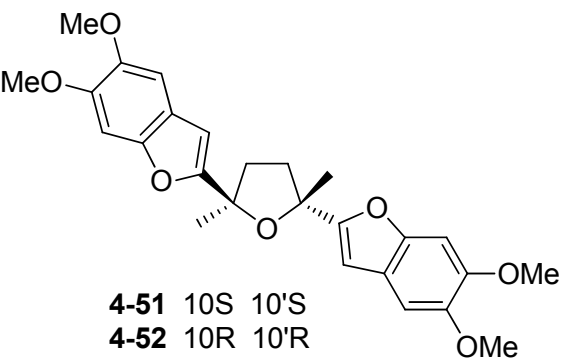

\section{照 Springer}


1.4 Others: Besides the above terpenoid constituents, there were still one steroid (4-1), 11 alkaloids (4-2 to 4-12), two flavonoids (4-13 and 4-14), and three lignans (4-15 to 4-17), as well as other secondary metabolites, possessing various

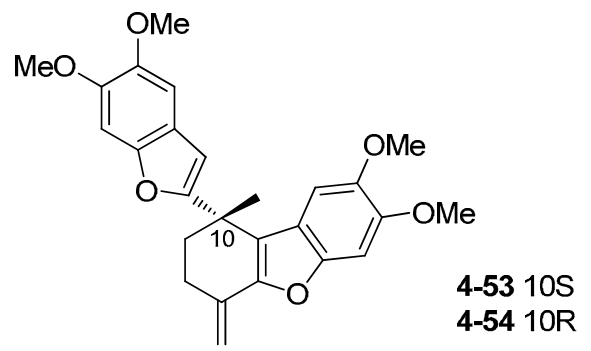<smiles></smiles>

4-56<smiles>CCC1C[C@](C)(C(C)(O)C(=O)OC)OC1=O</smiles>

4-60<smiles>C=C(C)c1cc2cc(C(C)=O)c(O)cc2o1</smiles>

4-61<smiles>C=C(CC(C)(C)CC(C)(C)c1cc2cc(OC)c(OC)cc2o1)c1cc2cc(OC)c(OC)cc2o1</smiles>

4-55<smiles>COc1cc2c(cc1OC)C(=O)C[C@@](C)(c1cc3cc(OC)c(OC)cc3o1)O2</smiles><smiles>C=C(CC(C)(C)C(=O)Oc1cc(C=O)c(OC)cc1OC)c1cc2cc(OC)c(OC)cc2o1</smiles>

4-58<smiles>O=C(/C=C/c1ccc(O)c(O)c1)O[C@H]1C[C@H](O)C[C@](O)(C(=O)O)[C@H]1OC(=O)/C=C/c1ccc(O)c(O)c1</smiles>

4-63<smiles>CC(C)=[W]C(C)(C)C</smiles><smiles>C=C(C)c1cc2cc(C(C)=O)c(OC)cc2o1</smiles>

4-62

Table 1. Sesquiterpenoids from the genus Ligularia

\begin{tabular}{|c|c|c|c|c|}
\hline No. & compound name & plant source & part of plant & Ref. \\
\hline 1-1 & $(6 \alpha, 8 \alpha)$-6-Hydroxyeremophil-7(11)-en-12,8-olide & L. muliensis & root & [15] \\
\hline 1-2 & $(6 \alpha, 8 \alpha)$-6-Acetyloxyeremophil-7(11)-en-12,8-olide & L. muliensis & root & [15] \\
\hline $1-3$ & $3 \beta$-Aacetyloxy- $6 \beta$-(2-methylbutanoyloxy)-10 $\beta$-hydroxyeremophil-7(11)-en-12,8 $\alpha$-olide & L. japonica & root & [16] \\
\hline $1-4$ & $6 \beta$-Angeloyloxy-1 $\alpha, 8 \beta, 10 \beta$-Trihydroxyeremophil-7(11)-en- $12,8 \alpha$-olide & L. virgaurea & root & [17] \\
\hline $1-5$ & $6 \beta$-Angeloyloxy- $1 \beta, 10 \beta$-epoxy- $8 \beta$-ethoxyeremophil-7(11)-en- $12,8 \alpha$-olide & L. virgaurea & root & [17] \\
\hline
\end{tabular}




\begin{tabular}{|c|c|c|c|c|}
\hline 1-6 & $(6 \beta, 8 \alpha)$-6-Acetyloxy-8-hydroxyeremophil-7(11)-en-12,8-olide & L. muliensis & root & [15] \\
\hline 1-7 & No Name & L. veitchiana & root & [18] \\
\hline 1-8 & $1 \beta, 10 \beta$-Epoxy- $6 \beta$-acetoxy- $3 \beta$-angeloyloxy- $8 \beta$-hydroxy-eremophil-7(11)-en- $8,12 \alpha$-olide & L. sagitta & aerial part & [19] \\
\hline $1-9$ & $3 \beta$-Acetoxy-10 $\beta$-hydroxy- $6 \beta, 8 \beta$-dimethoxy - eremophil-7(11)-en- $12,8 \alpha$-olide & L. duciformis & whole plant & [20] \\
\hline 1-10 & $3 \beta$-Acetoxy- $6 \beta, 8 \beta, 10 \beta$-trihydroxyeremophil-7(11)-en- $12,8 \alpha$-olide & L. duciformis & whole plant & [20] \\
\hline 1-11 & $8 \beta$-Hydroxy- $6 \beta$-angeloyloxyeremophil-7(11)-en- $8 \alpha, 12$-olide- 15 -oic acid & L. przewalskii & root & [21] \\
\hline 1-12 & $(1 \beta, 3 \beta, 6 \beta, 8 \beta, 10 \beta)$-3-Angeloyloxy-1,10-epoxy-8-ethoxy-6-hydroxyeremophil-7(11)-en-8,12 $\alpha$-olide & L. sagitta & aerial part & [22] \\
\hline 1-13 & $1 \beta, 10 \beta$-Epoxy- $8 \beta$-ethoxy- $6 \beta$-((2'-methylacryloyl)oxy)eremophil-7(11)-en-12,8 $\alpha$-olide & L. virgaurea & root & [17] \\
\hline 1-14 & $\begin{array}{l}(3 \beta, 6 \beta, 8 \beta, 10 \beta)-3 \text {-Acetyl-8,10-dihydroxy-6-(2-methyl-1-oxobutoxy)eremophil-7(11)-eno-12,8- } \\
\text { lactone }\end{array}$ & L. fischeri & root & [23] \\
\hline 1-15 & $8 \beta, 10 \beta$-Dihydroxy- $6 \beta$-isobutyryloxyeremophil-7(11)-en-12,8-olide & L. kanaitzensis & root & [24] \\
\hline 1-16 & $(1 \beta, 3 \beta, 6 \beta, 8 \beta, 10 \beta)$-6-Acetoxy-3-angeloyloxy-1,10-epoxy-8-hydroxyeremophil-7(11)-en- $8,12 \alpha$-olide & L. sagitta & aerial part & [22] \\
\hline 1-17 & $(1 \beta, 3 \beta, 6 \beta, 8 \beta, 10 \beta)$-3-Angeloyloxy-1,10-epoxy-6,8-dihydroxyeremophil-7(11)-en- $8,12 \alpha$-olide & L. sagitta & aerial part & [22] \\
\hline 1-18 & $(1 \beta, 3 \beta, 8 \beta, 10 \beta)$-3-Angeloyloxy)-1,10-epoxy-8-hydroxyeremophil-7(11)-en-8,12 $\alpha$-olide & L. sagitta & aerial part & [22] \\
\hline 1-19 & (-)-6-Oxoeremophilenolide & $\begin{array}{l}\text { L. fischeri var. } \\
\text { spiciformis }\end{array}$ & leaves & [25] \\
\hline $1-20$ & $6 \beta, 8 \beta$-Dimethoxy-10 $\beta$-hydroxyeremophil-7(11)-en-12,8 $\alpha$-olide & L. sagitta & root & [26] \\
\hline $1-21$ & $6 \beta$-Angeloyloxy- $10 \beta$-hydroxy- $8 \beta$-methoxy-eremophil-7(11)-en- $12,8 \alpha$-olide & L. sagitta & root & [26] \\
\hline 1-22 & $1 \beta, 10 \beta$-Epoxy- $6 \beta, 8 \beta$-dihydroxy-eremophil-7(11)-en- $12,8 \alpha$-olide & L. veitchiana & whole plant & [27] \\
\hline $1-23$ & $6 \beta$-(2'-Methylbutanoyloxy)-10 $\beta$-hydroxy- $8 \beta$-methoxyeremophil-7(11)-en-12,8 $\alpha$-olide & L. sagitta & root & [26] \\
\hline $1-24$ & $\begin{array}{l}1 \beta, 10 \beta \text {-Epoxy- } 6 \beta \text {-(2'-semialdehydeacetal-hydroxymethylacryloyloxy)- } 8 \beta \text {-ethoxy-eremophil-7(11)- } \\
\text { en-12,8 } \alpha \text {-olide }\end{array}$ & L. veitchiana & whole plant & [28] \\
\hline $1-25$ & $1 \beta, 10 \beta$-Epoxy-6 $\beta\left(2^{\prime}\right.$-hydroxymethylacryloyloxy)- $8 \beta$-hydroxy-eremophila-7(11)-en-12,8 $\alpha$-olide & L. veitchiana & root & [29] \\
\hline $1-26$ & $1 \beta, 10 \beta$-Epoxy- $6 \beta(2$-hydroxymethylacryloyloxy)- $8 \beta$-ethoxy-eremophila-7(11)-en- $12,8 \alpha$-olide & L. veitchiana & root & [29] \\
\hline $1-27$ & $1 \beta, 6 \beta$-Diangeloyloxy- $8 \beta, 10 \beta$-dihydroxyeremophil-(11)-en- $8 \alpha, 12$-olide & L. myriocephala & whole plant & [30] \\
\hline 1-28 & $1 \beta$-Angeloyloxy- $6 \beta, 10 \beta$-dihydroxy- $8 \beta$ - methoxyeremophila-7(11)-en- $8 \alpha, 12$-olide & L. myriocephala & whole plant & [13] \\
\hline 1-29 & $6 \beta, 8 \beta$-Diacetyl-2-oxoeremophila-1(10),7(11)-dien-12,8-olide & $\begin{array}{l}\text { L. virgaurea spp. } \\
\text { oligocephala }\end{array}$ & whole plant & [31] \\
\hline 1-30 & $3 \beta$-Angeloyloxy- $6 \beta$-hydroxy- $8 \beta$-methoxyeremophil-7(11)-en- $12,8 \alpha$-olide & L. hiberniflorum & rhizome & [32] \\
\hline 1-31 & $1 \beta, 10 \beta$-Epoxy- $6 \beta$-hydroxy- $8 \beta$-methoxy-eremophil-7(11)-en- $12,8 \alpha$-olide & L. veitchiana & root & [33] \\
\hline 1-32 & $1 \beta, 10 \beta$-Epoxy- $3 \beta$-acetoxy- $6 \beta$-angeloyloxy- $8 \beta$-hydroxy-eremophil-7(11)-en- $12,8 \alpha$-olide & L. veitchiana & root & [33] \\
\hline 1-33 & $1 \beta, 10 \beta$-Epoxy- $6 \beta$-(2'-hydroxymethylacrylyloxy)- $8 \beta$-methoxy-eremophil-7(11)-en- $12,8 \alpha$-olide & L. veitchiana & root & [33] \\
\hline 1-34 & $1 \beta, 10 \beta$-Epoxy- $6 \beta$-(2'-methoxymethylacrylyloxy)- $8 \beta$-hydroxy-eremophil-7(11)-en-12,8 $\alpha$-olide & L. veitchiana & root & [33] \\
\hline $1-35$ & $1 \beta, 10 \beta$-Epoxy- $3 \beta$-acetoxy- $6 \beta$-(2'-methylacrylyloxy)- $8 \beta$-hydroxy-eremophil-7(11)-en- $12,8 \alpha$-olide & L. veitchiana & root & [33] \\
\hline $1-36$ & Subspicatin D & L. subspicata & root & [34] \\
\hline $1-37$ & $3 \beta$-Acetoxy- $6 \beta$-methoxyeremophila-7(11),9(10)-dien-12,8 $\beta$-olide & L. duciformis & whole plant & [20] \\
\hline 1-38 & $6 \beta$-Methoxyeremophil-7(11)-en- $8 \beta, 12$-olide & L. virgaurea & root & [35] \\
\hline 1-39 & $6 \beta$-(2'-Methylbutanoyloxy)-3 $\beta$-acetoxy- $10 \beta$-hydroxy- $8 \alpha$-methoxyeremophil-7(11)-en- $8 \beta, 12$-olide & L. hodgsonii & $\begin{array}{l}\text { root and } \\
\text { aerial part }\end{array}$ & [36] \\
\hline $1-40$ & $\begin{array}{l}(4 S, 5 S, 6 R, 8 R, 10 R) \text {-6-Angeloyloxy-8-hydroxyeremophil-7(11)-en-8,12-olide-15-carboxylic acid } \\
\text { methyl ester }\end{array}$ & L. hodgsonii & $\begin{array}{l}\text { root and } \\
\text { rhizome }\end{array}$ & [37] \\
\hline $1-41$ & $(4 S, 5 S, 6 R, 8 R, 10 R)$-6-Angeloyloxy-8-ethoxyeremophil-7(11)-en-8,12-olid-15-oic acid & L. hodgsonii & $\begin{array}{l}\text { root and } \\
\text { rhizome }\end{array}$ & [37] \\
\hline $1-42$ & $(4 S, 5 S, 6 S, 8 R, 10 R)$-6-Angeloyloxy-8-ethoxyeremophil-7(11)-en-8,12-olid-15-oic acid & L. hodgsonii & $\begin{array}{l}\text { root and } \\
\text { rhizome }\end{array}$ & [37] \\
\hline $1-43$ & $(3 \beta, 6 \beta, 8 \alpha, 10 \beta)$-3-Acetyl-6,8,10-trihydroxyeremophil-7(11)-eno-12,8-lactone & L. fischeri & root & [23] \\
\hline $1-44$ & 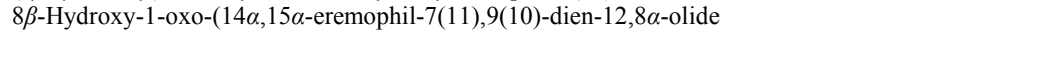 & L. platyglossa & $\begin{array}{l}\text { root and } \\
\text { rhizome }\end{array}$ & [38] \\
\hline $1-45$ & $3 \beta$-Acetoxy- $8 \alpha$-hydroxy- $6 \beta$-methoxyeremophila-7(11),9(10)-dien- $12,8 \beta$-olide & L. duciformis & whole plant & [20] \\
\hline $1-46$ & $3 \beta$-Acetoxy- $10 \beta$-hydroxy- $6 \beta, 8 \alpha$-di-methoxyeremophil-7(11)-en- $8 \beta, 12$-olide & L. hodgsonii & $\begin{array}{l}\text { root and } \\
\text { aerial part }\end{array}$ & [36] \\
\hline $1-47$ & $8 \alpha$-Methoxy- $6 \beta$-angeloyloxyeremophil-7(11)-en- $8 \beta, 12$-olide-14-oic acid & L. dolichobotrys & whole plant & [39] \\
\hline 1-48 & $3 \alpha, 4 \alpha$-Epoxy- $6 \alpha$-(2'-methylacryloyl)oxy- $8 \alpha$-ethoxyeremophil-7(11)-en- $8 \beta, 12$-olide & L. virgaurea & root & [35] \\
\hline $1-49$ & $6 \beta$-(2'-Methylbutanoyloxy)-3 $\beta$-acetoxy- $10 \beta$-hydroxyeremophil-7(11)-en- $8 \beta, 12$-olide & L. hodgsonii & $\begin{array}{l}\text { root and } \\
\text { aerial part }\end{array}$ & [36] \\
\hline 1-50 & $3 \alpha, 4 \alpha$-Epoxy- $6 \alpha$-(2'-methylacryloyl)oxy- $8 \alpha$-methoxyeremophil-7(11)-en- $8 \beta, 12$-olide & L. virgaurea & root & [35] \\
\hline 1-51 & $6 \beta$-(2'-Methylbutanoyloxy)- $10 \beta$-hydroxy- $8 \alpha$-methoxyeremophil-7(11)-en- $12,8 \beta$-olide & L. sagitta & root & [26] \\
\hline $1-52$ & $6 \beta$-Angeloyloxy-10 $\beta$-hydroxy- $8 \alpha$-methoxyeremophil-7(11)-en-12,8 $\beta$-olide & L. sagitta & root & [26] \\
\hline $1-53$ & $6 \beta, 8 \alpha$-Dihydroxy-1-oxoeremophila-7(11),9(10)-diene-12,8-olide & $\begin{array}{l}\text { L. virgaurea spp. } \\
\text { oligocephala }\end{array}$ & whole plant & [9] \\
\hline 1-54 & $1 \beta$-Angeloyloxy- $6 \beta, 10 \alpha$-dihydroxy- $8 \alpha$-methoxyeremophila-7(11)-en- $8 \beta, 12$-olide & L. myriocephala & whole plant & [13] \\
\hline $1-55$ & $3 \beta$-Angeloyloxy- $6 \beta, 8 \alpha$-dimethoxy-eremophil-7(11)-en- $12,8 \beta$-olide & L. hiberniflorum & rhizome & [32] \\
\hline $1-56$ & $1 \beta, 6 \beta$-Diangeloyloxy- $8 \alpha, 10 \alpha$-dihydroxy-eremophil-7(11)-en- $8 \beta, 12$-olide & L. myriocephala & whole plant & [30] \\
\hline $1-57$ & $3 \beta$-Angeloyloxy- $6 \beta$-hydroxy- $8 \alpha$-methoxyeremophil-7(11)-en- $12,8 \beta$-olide & L. hiberniflorum & rhizome & [32] \\
\hline $1-58$ & 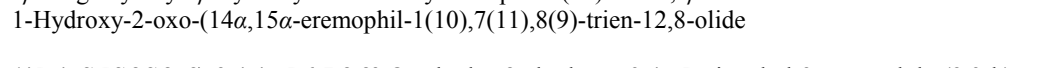 & L. platyglossa & $\begin{array}{l}\text { root and } \\
\text { rhizome }\end{array}$ & [38] \\
\hline $1-59$ & $\begin{array}{l}(4 R, 4 \mathrm{a} S, 5 S, 8 S, 8 \mathrm{a} S)-2,4,4 \mathrm{a}, 5,6,7,8,8^{\mathrm{a}} \text {-Octahydro-8a-hydroxy-3,4a,5-trimethyl-2-oxonaphtho(2,3-b) } \\
\text { furan-4,8-diyl diacetate. }\end{array}$ & L. sagitta & root & [40] \\
\hline 1-60 & No Name & L. veitchiana & root & [18] \\
\hline 1-61 & $3 \beta$-Acetyloxy- $6 \alpha$-hydroxyligularenolide & L. przewalskii & root & [41] \\
\hline $1-62$ & $6 \beta$-Angeloyloxy-2-oxoeremophil-1(10),7(11),8-triene-12,8-olide & L. virgaurea & root & [42] \\
\hline $1-63$ & $3 \beta$-Acetoxy- $10 \beta$-hydroxy- $6 \beta$-methoxy-eremophil-7(11),8(9)-dien-8,12-olide & L. hodgsonii & $\begin{array}{l}\text { root and } \\
\text { aerial part }\end{array}$ & [36] \\
\hline 1-64 & $6 \beta$-(2E-Methylbutyryloxy)eremophil-3,7(11),8-trien-8,12-olide-15-oic acid methyl ester & L. lapathifolia & root & [5] \\
\hline 1-65 & $(3 \beta, 6 \beta, 10 \beta)$-3-Acetyl-6,10-dihydroxy-eremophila-7(11),8-dieno-12,8-lactone & L. fischeri & root & [23] \\
\hline $1-66$ & $(3 \beta, 6 \beta, 10 \alpha)$-3-Acetyl-6,10-dihydroxy-eremophila-7(11),8-dieno-12,8-lactone & L. fischeri & root & [23] \\
\hline
\end{tabular}

\section{黛 Springer}




\begin{tabular}{|c|c|c|c|c|}
\hline $1-67$ & $6 \alpha, 10 \alpha$-Dihydroxy-1-oxoeremophila-7(11),8(9)-dien-8,12-olide & $\begin{array}{l}\text { L. virgaurea spp. } \\
\text { oligocephala }\end{array}$ & whole plant & [43] \\
\hline $1-68$ & $6 \beta, 10 \alpha$-Dihydroxy-1-oxoeremophila-7(11),8(9)-dien-8,12-olide & $\begin{array}{l}\text { L. virgaurea spp. } \\
\text { oligocephala }\end{array}$ & whole plant & [43] \\
\hline $1-69$ & $10 \alpha$-Hydroxy-1-oxoeremophila-7(11),8(9)-dien-8,12-olide & $\begin{array}{l}\text { L. virgaurea spp. } \\
\text { oligocephala }\end{array}$ & whole plant & [43] \\
\hline $1-70^{a}$ & Virgauride & L. virgaurea & root & [44] \\
\hline $1-71^{\mathrm{a}}$ & Virgauride & L. virgaurea & root & [44] \\
\hline 1-72 & $6 \beta$-Acetyl-2-oxoeremophila-1(10),7(11),8(9)-trien-12,8-olide & $\begin{array}{l}\text { L. virgaurea spp. } \\
\text { oligocephala }\end{array}$ & whole plant & [31] \\
\hline $1-73$ & $6 \beta, 10 \beta$-Dihydroxyeremophila-7(11),8(9)-dien-12,8-olide & $\begin{array}{l}\text { L. virgaurea spp. } \\
\text { oligocephala }\end{array}$ & whole plant & [45] \\
\hline $1-74$ & Eremofarfugin $\mathrm{C}$ & L. kanaitzensis & root & [24] \\
\hline $1-75$ & $6 \beta$-Hydroxy-3 $\beta$-(2'-methylacryloyl)oxy-11 $\beta H$-eremophil-7-en-12,8-olide & L. virgaurea & root & [42] \\
\hline $1-76$ & Eremofarfugin D & L. vellerea & root & [46] \\
\hline $1-77$ & Eremofarfugin E & L. vellerea & root & [46] \\
\hline $1-78$ & $3 \beta$-(2'-Methylbutanoyloxy)- $8 \beta H$-eremophil-7(11)-ene- $12,8 \alpha(14,6 \alpha)$-diolide & L. tongolensis & root & [47] \\
\hline 1-79 & $3 \beta$-Angeloyloxy- $8 \beta$ H-eremophil-7(11)-ene- $12,8 \alpha(14 \beta, 6 \alpha)$-dioxide & L. lapathifolia & $\begin{array}{l}\text { root and } \\
\text { rhizome }\end{array}$ & [48] \\
\hline 1-80 & $(1 R, 4 S, 5 S, 6 R, 8 S, 10 R)$-1-Acetoxyeremophil-7(11)-en-6,15;8,12-diolide & L. hodgsonii & $\begin{array}{l}\text { root and } \\
\text { rhizome }\end{array}$ & [37] \\
\hline 1-81 & $8 \beta$-Methoxyeremophil-7(11)-en- $6 \alpha, 15 ; 8 \alpha, 12$-diolie & L. przewalskii & root & [21] \\
\hline 1-82 & $10 \beta$-Hydroxy- $8 \beta, 9 \beta$-epoxyeremophil-7(11)-en- $6 \alpha, 15 ; 8 \alpha, 12$-diolide & L. przewalskii & root & [21] \\
\hline $1-83$ & $3 \beta$-Angeloyloxy- $8 \beta H$-hydroxyeremophil-7(11)-ene- $12,8 \alpha(14 \beta, 6 \alpha)$-dioxide & L. lapathifolia & $\begin{array}{l}\text { root and } \\
\text { rhizome }\end{array}$ & [48] \\
\hline 1-84 & $(1 R, 4 S, 5 S, 6 R, 8 S, 10 R)$-1-Acetoxy- $8 \beta$-hydroxyeremophil-7(11)-en-6,15;8,12-diolide & L. hodgsonii & $\begin{array}{l}\text { root and } \\
\text { rhizome }\end{array}$ & [37] \\
\hline $1-85$ & $(4 S, 5 S, 6 R, 8 R, 9 S, 10 S)-8$-Hydroxy-9-(angeloyloxy)eremophil-7(11)-en-6,15;8,12-diolide & L. hodgsonii & $\begin{array}{l}\text { root and } \\
\text { rhizome }\end{array}$ & [37] \\
\hline $1-86$ & $3 \beta$-Angeloyloxy- $8 \beta H$-methoxyeremophil-7(11)-ene- $12,8 \alpha(14 \beta, 6 \alpha)$-dioxide & L. lapathifolia & $\begin{array}{l}\text { root and } \\
\text { rhizome }\end{array}$ & [48] \\
\hline 1-87 & $3 \beta$-Angeloyloxy- $8 \beta H$-ethoxyeremophil-7(11)-ene- $12,8 \alpha(14 \beta, 6 \alpha)$-dioxide & L. lapathifolia & $\begin{array}{l}\text { root and } \\
\text { rhizome }\end{array}$ & [48] \\
\hline 1-88 & $7 \alpha, 8 \alpha$-Epoxy-eremophil-an- $12 \beta, 8 \beta(14 \beta, 6 \alpha)$-diolide & L. intermedia & rhizome & [49] \\
\hline 1-89 & $8 \alpha$-Hydroxyeremophil-7(11)-ene-12,8$\beta(14 \beta, 6 \alpha)$-diolide & L. intermedia & rhizome & [50] \\
\hline $1-90$ & Eremophil-7(8)-en-12,8(14 $\beta, 6 \alpha)$-diolide & L. intermedia & rhizome & [49] \\
\hline 1-91 & Eremophil-8(9),7(11)-dien-6 $\alpha, 15 ; 8,12$-diolide & L. przewalskii & root & [21] \\
\hline $1-92$ & $10 \beta$-Hydroxyeremophil-8(9),7(11)-dien-6 $\alpha, 15 ; 8,12$-diolide & L. przewalskii & root & [21] \\
\hline $1-93$ & $(4 S, 5 S, 6 R, 10 R)$-10-Hydroxyeremophil-7(11),8(9)-diene-6,15;8,12-diolide & L. hodgsonii & $\begin{array}{l}\text { root and } \\
\text { rhizome }\end{array}$ & [37] \\
\hline $1-94$ & $3 \beta$-Angeloyloxy-10 $\beta$-hydroxyeremophil- $8(9), 7(11)$-diene-12,8(14 $\beta, 6 \alpha)$-dioxide & L. lapathifolia & $\begin{array}{l}\text { root and } \\
\text { rhizome }\end{array}$ & [48] \\
\hline $1-95$ & $8 \beta H$-Eremophil-3, $7(11)$-dien-12,8 $\alpha(14,6 \alpha)$-diolide & L. tongolensis & root & [47] \\
\hline $1-96$ & $8 \beta$-Hydroxyeremophil-3,7(11)-diene- $8 \alpha, 12(6 \alpha, 15)$-diolide & L. lapathifolia & root & [5] \\
\hline 1-97 & $8 \beta$-Methoxyeremophil-3,7(11)-diene-8R,12(6R,15)-diolide & L. lapathifolia & root & [5] \\
\hline $1-98$ & $8 \beta$-Ethoxyeremophil-3,7(11)-diene- $8 \alpha, 12(6 \alpha, 15)$-diolide & L. lapathifolia & root & [5] \\
\hline 1-99 & $8 \alpha$-Hydroxy-eremophil-3,7(11)-dien-12,8$\beta(14,6 \alpha)$-diolide & L. atroviolacea & root & [51] \\
\hline 1-100 & $1 \alpha$-Acetoxyfuranoeremophilan-15,6 $\alpha$-olide & L. dictyoneura & root & [52] \\
\hline 1-101 & $3 \beta$-Angeloyloxyeremophilan-7,11-diene-14 $\beta, 6 \alpha$-olide & L. lapathifolia & $\begin{array}{l}\text { root and } \\
\text { rhizome }\end{array}$ & [48] \\
\hline 1-102 & $3 \beta$-Angeloyloxy- 8,12 -epoxy- $12 \alpha$-hydroxy- $8 \beta$-methoxyeremophil-7(11)-en- $14 \beta, 6 \alpha$-olide & L. lapathifolia & $\begin{array}{l}\text { root and } \\
\text { rhizome }\end{array}$ & [48] \\
\hline 1-103 & Ligumacrophyllatin & L. macrophylla & root & [53] \\
\hline 1-104 & 9-Oxoplatyphyllide & L. fischeri & root & [54] \\
\hline 1-105 & $6 \alpha, 9$-Dihydroxy-14 $\beta$-carboxyfuranoeremophil-9(10)-ene & L. intermedia & rhizome & [49] \\
\hline $1-106$ & 1,3-Dimethoxy-4,6,11-trimethylnapbthofuran & L. przewalskii & root & [55] \\
\hline 1-107 & $6 \beta$-Angeloyloxy-furanoligularenone & L. pleurocaulis & $\begin{array}{l}\text { root and } \\
\text { rhizome }\end{array}$ & [56] \\
\hline 1-108 & $6 \beta$-Acetoxyfuranoeremophilan- $10 \beta$-ol & L. kanaitzensis & root & [24] \\
\hline 1-109 & Subspicatin A & L. subspicata & root & [34] \\
\hline $1-110$ & Subspicatin B & L. subspicata & root & [34] \\
\hline 1-111 & Subspicatin C & L. subspicata & root & [34] \\
\hline 1-112 & $6 \beta$-Angeloyloxyfuranoeremophil-1(10)-en-2-one & L. virgaurea & root & [42] \\
\hline $1-113$ & $6 \beta$-Acetoxyfuranoeremophil-1(10)-en-2-one & L. virgaurea & root & [42] \\
\hline 1-114 & $6 \beta$-Hydroxyfuranoeremophil-1(10)-en-2-one & L. virgaurea & root & {$[42]$} \\
\hline 1-115 & $\begin{array}{l}\text { ( } 4 S, 4 \mathrm{a} S, 5 S, 8 R, 8 \mathrm{a} S)-4,4 \mathrm{a}, 5,6,7,8,8 \mathrm{a}, 9-O c t a h y d r o-8,8 \mathrm{a} \text {-dihydroxy-3,4a,5-trimethylnaphtho(2,3- } \\
\text { b)furan-4-yl(2-(hydroxymethyl)prop-2-enoate }\end{array}$ & L. sagitta & root & [40] \\
\hline 1-116 & $1 \alpha$-Chloro- $6 \beta$-isobutyroxy-9-oxo- $10 \beta$-hydroxy-furanoeremophilane & L. atroviolacea & root & [57] \\
\hline 1-117 & $6 \beta$-Sarracinoyloxy-1 $\beta, 10 \beta$-epoxy-furanoeremophilane & L. macrophylla & $\begin{array}{l}\text { root and } \\
\text { rhizome }\end{array}$ & [58] \\
\hline 1-118 & 6 $\beta$-(Z-4'-Hydroxy-2-methyl-2-butenoyl)oxyfuranoeremophil-1(10)-en-2-one & L. virgaurea & root & [42] \\
\hline 1-119 & $6 \alpha$-Angeloyloxy-10 $\beta H$-furanoeremophil-1-one & L. macrophylla & $\begin{array}{l}\text { root and } \\
\text { rhizome }\end{array}$ & [58] \\
\hline 1-120 & $1 \alpha$-Hydroxy-9-deoxycacalol & L. macrophylla & $\begin{array}{l}\text { root and } \\
\text { rhizome }\end{array}$ & [58] \\
\hline 1-121 & $3 \beta$-Acetoxy- $6 \beta$-(angeloyloxy)furanoeremophilan- $10 \beta$-ol & L. dictyoneura & root & [52] \\
\hline $1-122$ & Franchetianone B & L. franchetiana & root & [59] \\
\hline
\end{tabular}




\begin{tabular}{|c|c|c|c|c|}
\hline 1-123 & $6 \beta$-(2-(Hydroxymethyl)prop-2-enoyloxy)furanoeremophil-1(10)-ene & L. dictyoneura & root & [52] \\
\hline $1-124$ & $1 \beta, 10 \alpha$-Dihydroxy- $6 \beta-((2$-methylpropyl)oxy)furanoeremophil-9-one & $\begin{array}{l}\text { L. virgaurea spp. } \\
\text { oligocephala }\end{array}$ & whole plant & {$[1]$} \\
\hline $1-125$ & $3 \beta$-Acetoxy-6 $\beta$-(2-methylbutyryloxy)furanoeremophilan- $10 \beta$-ol & L. oligonema & root & [60] \\
\hline 1-126 & $3 \beta$-Acetoxy- $6 \beta$-isobutyryloxyfuranoeremophilan- $10 \beta$-ol & L. oligonema & root & [60] \\
\hline 1-127 & Furanoeremophil-1(10)-en- $6 \alpha$-ol & L. anoleuca & root & [61] \\
\hline 1-128 & Benzofuranoeremophil-2-ene & L. sagitta & rhizome & [62] \\
\hline 1-129 & 1,3-Dimethoxy-4,6-dimethylnaphthofuran & L. veitchiana & root & [4] \\
\hline 1-130 & Eremoligularin & L. muliensis & root & [63] \\
\hline 1-131 & Subspicatolide & L. subspicata & root & [34] \\
\hline 1-132 & Franchetianone A & L. franchetiana & root & [59] \\
\hline 1-133 & $1 \beta$-Hydroxy- $6 \alpha$-isobutyryloxy-9-noreremophil-7(11),8(10)-dien-8(12)-olide & L. fischeri & root & [54] \\
\hline 1-134 & $1 \beta$-Acetoxy-6 $\alpha$-isobutyryloxy-9-noreremophil-7(11),8(10)-dien-8(12)-olide & L. fischeri & root & [54] \\
\hline 1-135 & Ligulactone A & L. fischeri & root & [14] \\
\hline 1-136 & Ligulactone B & L. fischeri & root & [14] \\
\hline 1-137 & Eremophila-1(10),7(11),8-triene-12,8-lactam & L. fischeri & root & [3] \\
\hline 1-138 & No Name & L. veitchiana & root & [18] \\
\hline 1-139 & $1 \beta, 11$-Dihydroxy-eremophil-9-ene & L. fischeri & whole plant & [64] \\
\hline $1-140$ & Ligudicin A & L. dictyoneura & $\begin{array}{l}\text { root and } \\
\text { rhizome }\end{array}$ & [65] \\
\hline 1-141 & 11-Hydroxy-eremophil 1(10)-en-2,9-dione & L. fischeri & whole plant & [64] \\
\hline 1-142 & $7 \alpha$-Hydroxy-9-en-1,8-dioxo-6,7-dihydroxyfuranoeremophilane & L. veitchiana & whole plant & [27] \\
\hline $1-143$ & 8-(( $\beta$-D-Glucopyranosyl)oxy)eremophila-1(10),8,11-trien-2-one & $\begin{array}{l}\text { L. virgaurea spp. } \\
\text { oligocephala }\end{array}$ & whole plant & [66] \\
\hline 1-144 & (7 $\alpha, 9 \alpha, 10 \alpha)$-9,10-Epoxy-eremophilan-11-ol & L. veitchiana & rhizome & [67] \\
\hline 1-145 & $3 \alpha$-Tigloyloxyeremophila-9,11-dien-8-one & L. kanaitzensis & root & [24] \\
\hline 1-146 & $3 \alpha$-Propionyloxy-7 $\beta H$-eremophila-9,11-dien-8-one & L. kanaitzensis & root & [24] \\
\hline 1-147 & $\begin{array}{l}(4 \mathrm{a} S, 5 S, 8 R)-5,6,7,8 \text {-Tetrahydro-8-hydroxy-3-(1-hydroxypropan-2-yl)-4a,5-dimethyl-naphthalen- } \\
2(4 \mathrm{a} H) \text {-one }\end{array}$ & L. sagitta & root & [40] \\
\hline 1-148 & $1 \beta$-Acetoxy-11(S)-8-oxoeremophil-6,9-dien-12-al & L. sagitta & root & [68] \\
\hline 1-149 & $1 \beta$-Acetoxy-11(R)-8-oxoeremophil-6,9-dien-12-al & L. sagitta & root & [68] \\
\hline 1-150 & $1 \beta, 12$-Diacetoxy-6,9,12E-trien-8-oxoeremophilane & L. sagitta & root & [68] \\
\hline 1-151 & $3 \beta$-Angeloyloxy-8-oxoeremophil-6(7)-ene-12,15-dioic acid methyl ester & L. lapathifolia & root & [5] \\
\hline 1-152 & Eremophil-6-en-11-ol & L. veitchiana & rhizome & [67] \\
\hline 1-153 & 11(R)-8-Oxoeremophil-6(7)-en-dimethyl-12,15-dioate & L. przewalskii & root & [21] \\
\hline 1-154 & 11(S)-8-Oxoeremophil-6(7)-en-dimethyl-12,15-dioate & L. przewalskii & root & [21] \\
\hline 1-155 & $3 \beta$-Acetyloxy-11-methoxy-8-oxoeremophila-6,9-dien-12-oic acid & L. przewalskii & root & [41] \\
\hline 1-156 & Kanaitzensol & L. kanaitzensis & root & [24] \\
\hline $1-157$ & $1 \beta$-Hydroxy-11(R,S)-8-oxoeremophil-6,9-dien-12-al & L. macrophylla & $\begin{array}{l}\text { root and } \\
\text { rhizome }\end{array}$ & [58] \\
\hline 1-158 & $1 \beta, 10 \beta$-Epoxy-7(11)-en-6 $\alpha$-hydroxy-8-oxo-eremophil-12-oic acid & L. sagitta & root & [69] \\
\hline 1-159 & $3 \beta$-Acetyloxy-11-(2'-methylbutanoyloxy)-8-oxoeremophila-6,9-dien-12-oic acid & L. przewalskii & root & [41] \\
\hline $1-160$ & (1 $\alpha$ )-1-Hydroxy-8-oxo-eremophila-6,9-dien-12-oic acid & $\begin{array}{l}\text { L. virgaurea spp. } \\
\text { oligocephala }\end{array}$ & whole plant & [66] \\
\hline 1-161 & 1 $\beta$-Angeloyloxy-8-oxoeremophil-6,9-dien-12-oic acid Me ester & L. myriocephala & whole plant & [30] \\
\hline 1-162 & $3 \beta$-Acetoxy-8-oxoeremophil-6(7),9(10)-dien-12-oic methyl ester & L. hodgsonii & $\begin{array}{l}\text { root and } \\
\text { aerial part }\end{array}$ & [36] \\
\hline $1-163$ & $\begin{array}{l}\text { 2-Acetyl-8a-methyl-2-(2-methyl-but-2-enoyloxy)-6-oxo-1,2,3,4,4a,5,6,8a-octahydro-naphthalene-1- } \\
\text { carboxylic acid }\end{array}$ & L. lapathifolia & $\begin{array}{l}\text { root and } \\
\text { rhizome }\end{array}$ & [70] \\
\hline 1-164 & $1 \beta$-Hydroxy-6(7),9(10)-dien-8-oxo-eremophil-12-oic acid & L. veitchiana & whole plant & [28] \\
\hline 1-165 & $(1 R, 4 S, 4 \mathrm{a} R)$-6-Acetyl-1,2,3,4,4a,5-hexahydro-4,4a-dimethylnaphthalene-1,7-diyl diacetate & L. sagitta & root & [40] \\
\hline $1-166$ & $(4 \mathrm{a} S, 5 S, 8 R)-5,6,7,8$-Tetrahydro-3,8-dihydroxy-4a,5-dimethylnaphthalen-2(4a $H)$-one & L. sagitta & root & [40] \\
\hline $1-167$ & $(2 R, 5 R, 8 S, 8 \mathrm{a} R)-1,2,3,5,6,7,8,8^{\mathrm{a}}$-Octahydro-5-hydroxy-8,8a-dimethyl-3-oxonaphthalen-2-yl acetate & L. sagitta & root & [40] \\
\hline 1-168 & $3 \beta$-((2-Methylacryloyl)oxy)-8-oxo-12-noreremophil-6-en-11-one & L. virgaurea & root & [17] \\
\hline 1-169 & $9 \beta$-Hydroxy-8-oxo-12-noreremophil-6-en-11-one & L. virgaurea & root & [17] \\
\hline 1-170 & $3 \beta$-(3-Methylbutanoyloxy)-11-noroxoeremophila-6(7),9(10)-diene-8,11-dione & L. japonica & root & [16] \\
\hline 1-171 & $3 \beta$-Acetyloxy- $6 \alpha, 7 \alpha$-epoxy-11-noreremophil-9(10)-ene-8,11-dione & L. japonica & root & [16] \\
\hline 1-172 & $1 \beta$-Hydroxy-6,9-dien-8-oxoeremophil-11-nor-11-ketone & L. veitchiana & root & [68] \\
\hline $1-173$ & $1 \beta$-Acetoxy- $6 \alpha, 7 \alpha$-epoxy-9-en-8-oxoeremophil-11-nor-11-ketone & L. veitchiana & root & [68] \\
\hline 1-174 & $\begin{array}{l}\text { (1Z)- } 1-((5 R, 8 S, 8 \mathrm{a} R)-5 \text {-Acetoxy-6,7,8,8a-tetrahydro-8,8a-dimethyl-3-oxonaphthalen-2(1H)-ylidene) } \\
\text { ethyl acetate }\end{array}$ & L. sagitta & root & [40] \\
\hline 1-175 & $1 \beta$-Acetoxy-6,9-dien-8-oxoeremophil-11-nor-11-ketone & L. sagitta & root & [68] \\
\hline 1-176 & $3 \beta$-Hydroxy-11-noreremophila-6(7),9(10)-diene-8,11-dione & L. japonica & root & [16] \\
\hline 1-177 & Ligudentatin A & L. dentata & root & [71] \\
\hline 1-178 & Ligudentatin B & L. dentata & root & [71] \\
\hline 1-179 & $8 \alpha$-Hydroxyligudentatol & L. dentata & root & [72] \\
\hline 1-180 & $8 \alpha$-Hydroxyligujapone & L. dentata & root & [72] \\
\hline 1-181 & 7-Acetyl-1-hydroxy-4-methylene-1,2,3,4-tetrahydronaphthalene & L. duciformis & root & [73] \\
\hline 1-182 & $1 \beta$-Hydroxy-6(7),9(10)-dien-8-oxo-12-nor-11-hydroxy-eremophiladiene & L. veitchiana & whole plant & [28] \\
\hline 1-183 & $(4 \mathrm{a} S, 5 S)-5,6,7,8$-Tetrahydro-3-hydroxy-4a,5-dimethylnaphthalen-2(4a $H)$-one & L. fischeri & root & [74] \\
\hline 1-184 & $3 \beta$-Acetyloxy-7-hydroxynoreremophila-6,9-dien-8-one & L. przewalskii & root & [41] \\
\hline 1-185 & $8 \beta$-Hydroxy-2-dehydroxyliguhodgsonal & L. przewalskii & root & [41] \\
\hline 1-186 & Liguladentanorol & L. dentata & root & [72] \\
\hline 1-187 & $10 \beta$ H-6 $\beta$-Acetoxy- $7 \beta, 11 \beta$-epoxyeremophilan-8-one & L.lamarum & root & [75] \\
\hline 1-188 & $10 \beta$ H-6 $\beta$-Acetoxy-7a,11a-epoxyeremophilan-8-one & L.lamarum & root & [75] \\
\hline 1-189 & $10 \beta$ H- $8 a, 11$-Epidioxyeremophil-6-en- $8 \beta$-ol & L.lamarum & root & [75] \\
\hline
\end{tabular}

\section{包 Springer}




\begin{tabular}{|c|c|c|c|c|}
\hline 1-190 & 12-Hydroxy-6-oxo-6,7-secobakk-7(11)-en-8,12-olide & L. subspicata & root & [75] \\
\hline 1-191 & Ligudentatol & L. dentata & rhizome & [76] \\
\hline 1-192 & Ligularate & L. fischeri & root & [54] \\
\hline $1-193$ & $\begin{array}{l}\text { 2-Acetyl-3a-methyl-5-(2-methyl-but-2-enoyloxy)-3a,4,5,6,7,7a-hexahydro- } 1 H \text {-indene-4-carboxylic } \\
\text { acid }\end{array}$ & L. lapathifolia & $\begin{array}{l}\text { root and } \\
\text { rhizome }\end{array}$ & [70] \\
\hline 1-194 & 2-Acetyl-3a $\beta$-methyl-3a,6,7,7a $\beta$-tetrahydro- $1 H$-inden-4-oic acid methyl ester & L. lapathifolia & root & [5] \\
\hline 1-195 & 1-((3aR,4S,7aR)-3a,4,5,6,7a-Hexahydro-3a,4-dimethyl-1H-inden-2-yl)ethanone & L. virgaurea & root & [42] \\
\hline $1-196$ & 2-Acetyl-3 $\alpha, \beta$-methyl-3 $\alpha, 4,5,6,7,7 \mathrm{a}$-hexahydroinden- $4 \beta$-carboxylic methyl ester & L. przewalskii & root & {$[21]$} \\
\hline 1-197 & $(3 \mathrm{a} R, 4 R, 5 S, 7 \mathrm{a} S)$-2-Acetyl-3a,4,5,6,7,7a-hexahydro-7a-hydroxy- $1 H$-inden-5-yl acetate & L. fischeri & root & [23] \\
\hline 1-198 & $5 \beta$-Angeloyloxy-3a, $4,5,6,7,7 \mathrm{a}$-hexahydro- $3 \mathrm{a} \beta$-methyl- $1 H$-indene- $2,4 \beta$-dioic acid methyl ester & L. virgaurea & root & [35] \\
\hline 1-199 & Ligulasagitin A & L. sagitta & root & [77] \\
\hline 1-200 & Ligulasagitin B & L. sagitta & root & [77] \\
\hline 1-201 & Ligulasagitin $\mathrm{C}$ & L. sagitta & root & [77] \\
\hline $1-202$ & Ligulaverin A & L. veitchiana & whole plant & [78] \\
\hline $1-203$ & Ligulaverin B & L. veitchiana & whole plant & [78] \\
\hline $1-204$ & Ligulaverin C & L. veitchiana & whole plant & [78] \\
\hline $1-205$ & Ligulaverin D & L. veitchiana & whole plant & [78] \\
\hline $1-206$ & Ligulaverin E & L. veitchiana & whole plant & [78] \\
\hline 1-207 & No Name & L. sagitta & root & [2] \\
\hline $1-208$ & No Name & L. sagitta & root & [2] \\
\hline $1-209$ & No Name & L. sagitta & root & [2] \\
\hline $1-210$ & No Name & L. sagitta & root & [2] \\
\hline $1-211$ & Altaicalarin C & L. altaica & $\begin{array}{l}\text { root and } \\
\text { rhizome }\end{array}$ & [79] \\
\hline $1-212$ & $(1 \beta, 2 \beta, 3 \beta, 4 \alpha, 6 \beta)$-Bisabol-7(14)-ene-1,2,3,4,8,10,11-heptol 2,10-diangelate & L. cymbulifera & root & [80] \\
\hline 1-213 & $(1 \beta, 2 \beta, 3 \beta, 4 \beta, 6 \beta)-3,4$-Epoxybisabol-7(14)-ene-1,2,8,10,11-pentol 2,10-diangelate & L. cymbulifera & root & [80] \\
\hline $1-214$ & $2 \beta, 8$-Bisangeloyloxy-3 $\beta, 4 \beta, 10,11$-bisepoxybisabol-7(14)-en- $\beta$-ol & L. lankongensis & root & [81] \\
\hline $1-215$ & $2 \beta, 8$-Bisangeloyloxy- $4 \alpha$-chloro-10,11-epoxybisabol- $7(14)$-ene-1 $\beta, 3 \beta$-diol & L. lankongensis & root & [81] \\
\hline $1-216$ & $\begin{array}{l}(1 \beta, 2 \beta, 3 \beta, 4 \alpha, 6 \beta) \text {-Bisabol-7(14)-ene-1,2,3,4,8,10,11-heptol2,10-diangelate-4-((3S,5S,6S)- } \\
\text { tetrahydroclivonecate) }\end{array}$ & L. cymbulifera & root & [80] \\
\hline 1-217 & 10,11-Epoxy-1 $\beta$-hydroxy- $2 \beta, 4 \alpha, 8$-triangeloyloxybisabol-7(14)-ene & L. cymbulifera & root & [82] \\
\hline 1-218 & $2 \beta, 8$-Diangeloyloxy-3 $\alpha, 4 \alpha, 10,11$-diepoxy-1 $\alpha$-hydroxybisabol-7(14)-ene & L. cymbulifera & root & [82] \\
\hline 1-219 & $2 \alpha, 8$-Diangeloyloxy-3 $\beta, 4 \beta, 10,11$-diepoxy-1 $\alpha$-hydroxybisabol-7(14)-ene & L. cymbulifera & root & [82] \\
\hline 1-220 & $(1 \alpha, 2 \alpha, 3 \beta, 5 \alpha, 6 \beta)-2,5,8$-Tris(angeloyloxy)-10,11-epoxy-1,3-dihydroxybisabol-7(14)-en-4-one & L. dentata & root & [83] \\
\hline 1-221 & $\left(1 \alpha, 2 \beta, 3 \beta, 4 R^{*}, 5 \alpha, 6 \alpha\right)-3,6,9-$ Tris((angeloyl)oxy)bisabol-10(15)-ene-2,4,5,7,11-pentol & L. lankongensis & root & [84] \\
\hline $1-222$ & $\left(1 \alpha, 2 \beta, 3 \beta, 4 R^{*}, 5 \alpha, 6 \alpha\right)-3,6,7-$ Tris((angeloyl)oxy)bisabol-10(15)-ene-2,4,5,9,11-pentol & L. lankongensis & root & [84] \\
\hline $1-223$ & Altaicalarin D & L. altaica & $\begin{array}{l}\text { root and } \\
\text { rhizome }\end{array}$ & [79] \\
\hline 1-224 & Ligudentatone A & L. dentata & root & [85] \\
\hline 1-225 & Ligudentatone B & L. dentata & root & [85] \\
\hline $1-226$ & $4 \alpha$-Chloro-1 $\beta, 8$-diangeloyloxy-10,11-epoxy-2$\beta$-hydroxybisabol-7(14)-ene & L. cymbulifera & root & [82] \\
\hline $1-227$ & $1 \beta, 5 \alpha, 8$-Trisangeloyloxy- $\beta, 4 \beta, 10,11$-bisepoxybisabol-7(14)-en- $\beta$-ol & L. lankongensis & root & [81] \\
\hline $1-228$ & $3 \beta, 4 \beta, 10,11$-Diepoxy-1 $\beta, 2 \beta, 8$-triange-loyloxybisabol-7(14)-ene & L. cymbulifera & root & [82] \\
\hline 1-229 & 1 $\beta, 8$-Diangeloyloxy-3 $\beta, 4 \beta, 10,11$-diepoxybisabol-7(14)-ene & L. cymbulifera & root & [82] \\
\hline $1-230$ & $1 \beta, 8$-Diangeloyloxy-3 $\beta, 4 \beta$-epoxy- $2 \beta, 10,11$-trihydroxybisabol-7(14)-ene & L. cymbulifera & root & [82] \\
\hline $1-231$ & $1 \alpha, 8$-Diangeloyloxy-10,11-dihydroxy-3 $\beta, 4 \beta$-epoxybisabol-7(14)-en-2-one & L. cymbulifera & root & [82] \\
\hline $1-232$ & $\begin{array}{l}(1 S, 2 R, 6 S, 9 R)-2,7-B i s(\text { angeloyloxy)-6,9-epoxy-11-hydroxy-6-methoxybisabola-3,10(15)-dien-5- } \\
\text { one }\end{array}$ & L. dentata & root & [86] \\
\hline 1-233 & $(1 \alpha, 2 \alpha, 3 \beta, 5 \alpha, 6 \beta)-1,5,8$-Tris(angeloyloxy)-10,11-epoxy-2,3-dihydroxybisabol-7(14)-en-4-one & L. dentata & root & [83] \\
\hline $1-234$ & $\begin{array}{l}(1 \alpha, 2 \beta, 3 \beta, 5 \alpha, 6 \beta)-1,8 \text {-Bis(angeloyloxy)-2,3-epoxy-5,10-dihydroxy-11-metho-xybisabol-7(14)-en-4- } \\
\text { one }\end{array}$ & L. dentata & root & [83] \\
\hline 1-235 & 8-Angeloyloxy-3 $\beta, 4 \beta, 10,11$-bisepoxy-1 $\beta$-(2-methylbutyryloxy)bisabol-7(14)-en-2 $\beta$-ol & L. lankongensis & root & [81] \\
\hline 1-236 & $(1 E, 5 R)$-3-Acetoxy-9-angeloyloxy-5-hydroxybisabola-3,1(10),7(11)-trien-2-one & L. dentata & root & [86] \\
\hline 1-237 & 9-Angeloyloxy-11-methoxybisabola-1,3,5,10(15)-tetraene-5,6,7-triol & L. dentata & root & {$[86]$} \\
\hline 1-238 & $9 \alpha$-Angeloyloxy-7 $\beta, 10 \beta$-epoxy-11-methoxybisabola-1,3,5-triene-5,6-diol & L. dentata & root & [86] \\
\hline 1-239 & $(8 \beta, 10 \alpha)$-8-Angeloyloxy-5,10-epoxybisabola-1,3,5,7(14)-tetraene-2,4,11-triol & L. dentata & root & [83] \\
\hline $1-240$ & $(8 \beta, 10 \alpha)$-8-Angeloyloxy-5,10-epoxythiazolo(5,4-a)bisabola-1,3,5,7(14)-tetraene-4,11-diol & L. dentata & root & [83] \\
\hline $1-241$ & Altaicalarin A & L. altaica & $\begin{array}{l}\text { root and } \\
\text { rhizome }\end{array}$ & [79] \\
\hline 1-242 & Altaicalarin B & L. altaica & $\begin{array}{l}\text { root and } \\
\text { rhizome }\end{array}$ & [79] \\
\hline $1-243$ & $\begin{array}{l}(1 R, 5 S, 6 R, 7 S, 8 R, 9 S, 11 S)-4 \text {-Acetoxy-9-((4-acetoxy-4-methylsenecioyl)oxy)-8-((2- } \\
\text { methylbutanoyl)oxy)-11,12-epoxyoplop-10(14)-en-3-one }\end{array}$ & L. narynensis & root & [87] \\
\hline $1-244$ & $\begin{array}{l}(1 S, 3 \mathrm{a} R, 5 S, 6 R, 7 S, 7 \mathrm{a} R)-1 \text {-(1-Acetoxyethyl)octahydro-4-methylidene-7-((2S)-2-methyl-oxiran-2-yl)- } \\
\text { 6-(3-methylpentanoyl)oxy)-2-oxo- } 1 H \text {-inden-5-yl- }(2 E) \text {-4-acetoxy-3-methylpent-2-enoate }\end{array}$ & L. narynensis & root & [87] \\
\hline $1-245$ & $\begin{array}{l}(1 S, 2 R, 3 S, 3 \mathrm{a} R, 5 S, 6 R, 7 S, 7 \mathrm{a} S) \text {-2-Acetoxy-1-(1-acetoxyethyl)octahydro-3,6-bis( }(2 \text {-methylbutanoyl)- } \\
\text { oxy)-4-methylidene-7-((2S)-2-methyloxiran-2-yl)-1H-inden-5-yl( } 2 E) \text {-4-hydroxy-3-methylpent-2- } \\
\text { enoate }\end{array}$ & L. narynensis & root & [87] \\
\hline $1-246$ & $\begin{array}{l}(1 S, 2 R, 3 S, 3 \mathrm{a} R, 5 S, 6 R, 7 S, 7 \mathrm{a} S) \text {-2-Acetoxy-1-(1-acetoxyethyl)-octahydro-3,6-bis((2-methylbutanoyl)- } \\
\text { oxy)-4-methylidene-7-((2S)-2-methyloxiran-2-yl)-1H-inden-5-yl }(2 E) \text {-3-methylpent-2-enoate }\end{array}$ & L. narynensis & root & [87] \\
\hline $1-247$ & $\begin{array}{l}3 \beta, 4 \text {-Diacetoxy- } 9 \alpha \text {-(4-acetoxy-4-methyl-senecioyloxy)-2 } \beta, 8 \alpha \text {-di(2-methylbutyryl-oxy)-11 } \alpha, 12 \text { - } \\
\text { epoxyoplop-10,(14)-ene }\end{array}$ & L. narynensis & root & [88] \\
\hline $1-248$ & $\begin{array}{l}3 \beta, 4 \text {-Diacetoxy- } 8 \alpha \text {-(2-methylbutyryloxy)-9 } \alpha \text {-(4-methylsenecioyloxy)-11 } \alpha, 12 \text {-epoxyoplop-10,(14)- } \\
\text { ene }\end{array}$ & L. narynensis & root & [88] \\
\hline $1-249$ & $(5 \beta, 9 \beta)$-Guaia-6,10(14)-dien-9-ol & L. macrophylla & $\begin{array}{l}\text { root and } \\
\text { rhizome }\end{array}$ & [89] \\
\hline
\end{tabular}




\begin{tabular}{|c|c|c|c|c|}
\hline $1-250$ & $2 \alpha$-Hydroxy-1 $\beta H, 7 \alpha H, 10 \alpha H$-guai-4,11(12)-dien-3-one & L. narynensis & root & [88] \\
\hline $1-251$ & Liguducin A & L. duciformis & whole plant & [90] \\
\hline $1-252$ & Furanomexicanane-9-ene- 8 -one & L. virgaurea & root & [44] \\
\hline $1-253$ & $9 \beta, 10 \beta$-Epoxy-furanomexicanane- 8 -one & L. virgaurea & root & [44] \\
\hline 1-254 & $4 \alpha, 8 \beta, 9 \alpha$-Trihydroxy- $5 \alpha H-7(11)$-eudesmen- $12,8 \alpha$-olide & L. platyglossa & $\begin{array}{l}\text { root and } \\
\text { rhizome }\end{array}$ & [38] \\
\hline $1-255$ & Eudesma-4,11-diene-1 $\beta, 15$-diol & L. dentata & root & [86] \\
\hline $1-256$ & Liguducin B & L. duciformis & whole plant & {$[90]$} \\
\hline $1-257$ & $(+)$-Intermedeol & $\begin{array}{l}\text { L. fischeri var. } \\
\text { spiciformis }\end{array}$ & leaves & [25] \\
\hline 1-258 & Ligucyperonol & L. dentata & rhizome & [76] \\
\hline $1-259$ & 2-(3-Pentenyl)-3,7-dimethylbenzofuran-1,4-dione & L. virgaurea & rhizome & [91] \\
\hline $1-260$ & 1-Hydroxy-2-(3-pentenyl)-3,7-dimethylbenzofuran & L. virgaurea & rhizome & [91] \\
\hline $1-261$ & 1-Methoxy-2-(3-pentenyl)-3,7-dimethylbenzofuran & L. virgaurea & rhizome & {$[91]$} \\
\hline $1-262$ & Ligulolide A & $\begin{array}{l}\text { L. virgaurea spp. } \\
\text { oligocephala }\end{array}$ & whole plant & [9] \\
\hline $1-263$ & $(6 S)$ Ligulolide $\mathrm{C}$ & $\begin{array}{l}\text { L. virgaurea spp. } \\
\text { oligocephala }\end{array}$ & whole plant & [92] \\
\hline 1-264 & $(6 R)$ Ligulolide $\mathrm{C}$ & $\begin{array}{l}\text { L. virgaurea spp. } \\
\text { oligocephala }\end{array}$ & whole plant & [92] \\
\hline $1-265$ & Ligupersin A & L. persica & root & [93] \\
\hline $1-266$ & Ligupersin B & L. persica & root & [93] \\
\hline $1-267$ & Virgauronin & L. virgaurea & root & [94] \\
\hline $1-268$ & Bieremoligularolide & L. muliensis & root & {$[63]$} \\
\hline $1-269$ & $8 \beta$-(Eremophil-3',7'(11')-dien-12', $8^{\prime} \alpha ; 15^{\prime}, 6^{\prime} \alpha$-diolide)-eremophil-3,7(11)-dien-12,8 $\alpha ; 15,6 \alpha$-diolide & L. atroviolacea & root & {$[57]$} \\
\hline $1-270$ & Fischelactone & L. fischeri & root & {$[3]$} \\
\hline 1-271 & 8,8'-bi-3 $\beta$-Angeloyloxy-eremophil-7(11)-en-12,8 $\alpha(14 \beta, 6 \alpha)$-diolide & L. lapathifolia & $\begin{array}{l}\text { root and } \\
\text { rhizome }\end{array}$ & [95] \\
\hline 1-272 & Biligulaplenolide & L. platyglossa & $\begin{array}{l}\text { root and } \\
\text { rhizome }\end{array}$ & [38] \\
\hline $1-273$ & Virgaurol A & L. virgaurea & root & [96] \\
\hline $1-274$ & Virgaurol B & L. virgaurea & root & [96] \\
\hline $1-275$ & Ligulasagitin D & L. sagitta & root & {$[77]$} \\
\hline $1-276$ & Virgaurin A & L. virgaurea & root & [44] \\
\hline 1-277 & Ligularin A & $\begin{array}{l}\text { L. virgaurea } \text { spp. } \\
\text { oligocephala }\end{array}$ & whole plant & [1] \\
\hline $1-278$ & $\begin{array}{l}\text { 2-\{((5S)-5,6,7,8-Tetrahydro-9-hydroxy-3,5-dimethylnaphtho(2,3-b)furan-4-yl)methyl }\}-3,5- \\
\text { dimethyl-6-((3E)-pent-3-en-1-yl)-1-benzofuran-4,7-dione }\end{array}$ & L. virgaurea & rhizome & [97] \\
\hline 1-279 & $\begin{array}{l}(5 S)-5,6,7,7 \mathrm{a}, 7 \mathrm{~b}, 12 \mathrm{~b}-H e x a h y d r o-3,4,5,11,12 \mathrm{~b}-\text { pentamethy-10-((3E)-pent-3-en-1-yl)- } \\
\text { furo(3",2":6',7')-naphtho(1',8': 4,5,6)pyrano(3,2-b)benzofuran-9-ol }\end{array}$ & L. virgaurea & rhizome & [97] \\
\hline $1-280$ & Ligulolide D & $\begin{array}{l}\text { L. virgaurea } \text { spp. } \\
\text { oligocephala }\end{array}$ & whole plant & {$[1]$} \\
\hline 1-281 & Ligulolide B & $\begin{array}{l}\text { L. virgaurea spp. } \\
\text { oligocephala }\end{array}$ & whole plant & [31] \\
\hline 1-282 & Biliguhodgsonolide & L. hodgsonii & $\begin{array}{l}\text { root and } \\
\text { rhizome }\end{array}$ & [98] \\
\hline 1-283 & Ligulamulienin A & L. muliensis & rhizome & [99] \\
\hline 1-284 & Ligulamulienin B & L. muliensis & rhizome & [99] \\
\hline 1-285 & Virgaurol D & L. virgaurea & root & [96] \\
\hline $1-286$ & Virgaurol C & L. virgaurea & root & [96] \\
\hline $1-287$ & Ligulasagitin E & L. sagitta & root & [77] \\
\hline 1-288 & Ligulatrovine A & L. atroviolacea & root & {$[57]$} \\
\hline $1-289$ & Ligumacrophyllal & L. macrophylla & root & [53] \\
\hline
\end{tabular}

${ }^{\mathrm{a}}$ Compounds 1-70 and 1-71 were obtained as a pair of epimers at C-6, and both of them were named virgauride.

Table 2. Monoterpenoids and diterpenoids from the genus Ligularia

\begin{tabular}{|c|c|c|c|c|}
\hline No. & compound class and name & plant source & part of plant & Ref. \\
\hline 2-1 & $\begin{array}{l}\text { Monoterpenoids } \\
\text { rel- }(1 R, 2 R, 3 R, 4 S, 5 S) \text {-p-Menthane- } 1,2,3,5 \text {-tetrol }\end{array}$ & L. muliensis & root & [15] \\
\hline \multirow[t]{2}{*}{$2-2$} & $1 \alpha, 2 \beta, 3 \alpha, 6 \alpha$-Tetrahydroxy-p-menthane & L. narynensis & root & [88] \\
\hline & Diterpenoids & & & \\
\hline 2-3 & Sagittolactone & L. sagitta & rhizome & [62] \\
\hline $2-4$ & Spiciformisin A & L. fischeri var. spiciformis & leaves & [100] \\
\hline $2-5$ & Spiciformisin B & L. fischeri var. spiciformis & leaves & {$[100]$} \\
\hline
\end{tabular}

\section{第 Springer}


Table 3. Triterpenoids from the genus Ligularia

\begin{tabular}{|c|c|c|c|c|}
\hline No. & compound name & plant source & part of plant & Ref. \\
\hline 3-1 & $7 \beta, 16 \beta, 28$-Triacetoxyolean-12-en-3-one & L. sagitta & root & [40] \\
\hline 3-2 & 3 -oxo- $16 \beta$-Hydroxy-olean- 12 -ene- 28 -al & L. odontomanes & whole plant & [101] \\
\hline 3-3 & Liguveitoside B & L. veitchiana & root and rhizome & [102] \\
\hline $3-4$ & Liguveitoside A & L. veitchiana & whole plant & {$[103]$} \\
\hline 3-5 & 3,4-Seco-olean-12-en-4-ol-3,28-dioic acid & L. intermedia & root & [104] \\
\hline 3-6 & A-homo-3a-oxa-olean-12-en-3-one-28-oic acid & L. intermedia & root & [104] \\
\hline 3-7 & $2 \alpha, 3 \beta, 19 \alpha$-trihydroxy-28-norurs-12-ene & L. tongolensis & root & [47] \\
\hline 3-8 & $2 \alpha, 3 \alpha, 19 \alpha$-trihydroxy-28-norurs-12-ene & L. tongolensis & root & {$[47]$} \\
\hline 3-9 & Monocyclosqualene & L. fischeri var. spiciformis & leaves & [100] \\
\hline 3-10 & 24-Chlorocycloart-25-en-3 $\beta$-ol & L. stenocephala & root and leaves & [105] \\
\hline
\end{tabular}

Table 4. Other chemical constituents from the genus Ligularia

\begin{tabular}{|c|c|c|c|c|}
\hline No. & compound class and name & plant source & part of plant & Ref. \\
\hline & Steroids & & & \\
\hline 4-1 & $\begin{array}{l}3 \beta, 7 \alpha, 22 \text {-Trihydoxystigmast- } 5 \text {-ene } \\
\text { Alkaloids }\end{array}$ & L. dolichobotrys & whole plant & [39] \\
\hline 4-2 & $O$-Acetylyamataimine & L. tsangchanensis & root & [106] \\
\hline 4-3 & $O$-Acetylyamataimine $\mathrm{N}$-oxide & L. tsangchanensis & root & [106] \\
\hline 4-4 & Clivorine & L. hodgsonii & whole plant & {$[107]$} \\
\hline 4-5 & Ligularine & L. hodgsonii & whole plant & [107] \\
\hline 4-6 & Lankongensisine $\mathrm{A}$ & L. lankongensis & root & [108] \\
\hline 4-7 & Lankongensisine B & L. lankongensis & root & [108] \\
\hline 4-8 & 1-(( $\beta$-D-Glucopyranosyloxy)methyl)-5,6-dihydropyrrolizin-7-one & L. cymbulifera & root & [80] \\
\hline 4-9 & 3,9-Dimethyl-5-nitropyrido(3,2,1-ij)quinazoline-1,7-dione & L. duciformis & rhizome & [109] \\
\hline 4-10 & 1-(4'-Methylpyridazin-5'-yl)butane-1,2,3,4-tetraol & L. duciformis & rhizome & [109] \\
\hline 4-11 & 2,7-Bis(isopropylimino)- $2 \mathrm{H}, 7 \mathrm{H}$-dicyclopentacyclooctene-4,9-diol & L. duciformis & rhizome & [109] \\
\hline 4-12 & $\begin{array}{l}\text { N,N-Di(1-iminopropyl)propionamidine } \\
\text { Flavonoids }\end{array}$ & L. duciformis & rhizome & [109] \\
\hline 4-13 & 6-Acetyl-8-methoxy-2,3-dimethylchromen-4-one & L. macrophylla & root and rhizome & [89] \\
\hline 4-14 & $\begin{array}{l}(2 S) \text {-3'-Hydroxy-5',7-dimethoxyflavanone } \\
\text { Lignans }\end{array}$ & L. macrophylla & root and rhizome & [89] \\
\hline 4-15 & $9 \alpha$-Angeloyloxypinoresinol & L. kanaitizensis & root and rhizome & [110] \\
\hline 4-16 & 4-( $(\beta$-D-Glucopyranosyl)oxy)pinoresinol & L. virgaurea spp. oligocephala & whole plant & [66] \\
\hline 4-17 & $\begin{array}{l}\text { Narynenol } \\
\text { Others }\end{array}$ & L. narynensis & root & [111] \\
\hline 4-18 & 2-Isoprepenyl-6-acetyl-8-methoxy-1,3-benzodioxin-4-one & L. intermedia & root & [112] \\
\hline 4-19 & 6-Acetyl-7-hydroxy-2-isopropylidene-benzo $(1,4)$ dioxin-3-one & L. stenocephala & root & [113] \\
\hline 4-20 & 2,5-Dihydroxy-6,7-dimethylnaphthoquinone & L. vellerea & whole plant & [6] \\
\hline 4-21 & Ligumedial & L. intermedia & root and rhizome & [114] \\
\hline 4-22 & Ligumediaoic acid & L. intermedia & root and rhizome & [114] \\
\hline 4-23 & 7,8-Dimethoxy-1,4-dimethyldibenzofuran & L. stenocephala & root and leaves & [105] \\
\hline 4-24 & 1,2,4-Trimethyl-7,8-dimethoxy-dibenzofuran & L. caloxantha & root & [115] \\
\hline 4-25 & 4-O-(6-Hydroxy-7(9)-dehydro-6,7-dihydrogeranyl)-coniferyl alcohol & L. duciformis & root & [116] \\
\hline 4-26 & 4-O-(7-Hydroxy-5,6E-dehydro-6,7-dihydrogeranyl)-coniferyl alcohol & L. duciformis & root & [116] \\
\hline 4-27 & 4-O-(6-Hydroperoxy-7(9)-dehydro-6,7-dihydrogeranyl)-coniferyl alcohol & L. duciformis & root & [116] \\
\hline 4-28 & 4-O-(7-Hydroperoxy-5,6E-dehydro-6,7-dihydrogeranyl)-coniferyl alcohol & L. duciformis & root & [116] \\
\hline 4-29 & 4-O-(6-Hydroxy-7(9)-dehydro-6,7-dihydrogeranyl)-sinapyl alcohol & L. duciformis & root & [116] \\
\hline 4-30 & 4-O-(6-Hydroxy-7(9)-dehydro-6,7-dihydrogeranyl)-coniferyl alcohol & L. duciformis & root & [116] \\
\hline 4-31 & $(E, E)-4-(7-H y d r o p e r o x y-3,7-d i m e t h y l o c t a-2,5$-dienyloxy)-syringenin & L. intermedia & root & [112] \\
\hline 4-32 & (E)-4-(6-Hydroperoxy-3,7-dimethylocta-2,7-dienyloxy)-syringenin & L. intermedia & root & [112] \\
\hline 4-33 & No name & L. nelumbifolia & root & [117] \\
\hline 4-34 & No name & L. nelumbifolia & root & {$[117]$} \\
\hline 4-35 & No name & L. nelumbifolia & root & {$[117]$} \\
\hline 4-36 & 3,4,5-Trimethoxycinnamyl angelic acid ester & L. veitchiana & root & [4] \\
\hline 4-37 & 4-((3',4'-Dihydroxycinnamoyl)-oxy)-methyl cinnamate & L. vellerea & whole plant & [6] \\
\hline 4-38 & 2-Acetyl-5,6-dimethoxybenzofuran & L. przewalskii & root & {$[55]$} \\
\hline 4-39 & 2-Propenyl-5-acetyl-7-hydroxy-2,3-dihydrobenzofuran & L. przewalskii & root & {$[55]$} \\
\hline 4-40 & 5-Acetyl-7-methoxybenzofuran & L. przewalskii & root & [55] \\
\hline 4-41 & No name & L. nelumbifolia & root & [117] \\
\hline 4-42 & 6-Hydroxy-3 $\alpha$-methoxytremetone & L. stenocephala & root and leaves & [105] \\
\hline 4-43 & 2-Acetyl-5-isopentenyl-6-methylbenzofuran & L. veitchiana & root & [4] \\
\hline 4-44 & $\begin{array}{l}\text { 2,2'-(1",1"-Dimethyl-3"-methoxy-3"-methyl-1",3"-propanediyl)bis } \\
(5,6 \text {-dimethoxybenzofuran) }\end{array}$ & L. stenocephala & root and leaves & [105] \\
\hline 4-45 & Stenocephalin A & L. stenocephala & root & [118] \\
\hline 4-46 & Ligustenin $\mathrm{C}$ & L. stenocephala & root & [119] \\
\hline 4-47 & Ligustenin B & L. stenocephala & root & [119] \\
\hline 4-48 & Ligustenin D & L. stenocephala & root & [119] \\
\hline 4-49 & $(+)$-Ligulacephalin A & L. stenocephala & root & [120] \\
\hline $4-50$ & (-)-Ligulacephalin A & L. stenocephala & root & [120] \\
\hline 4-51 & $(+)$-Ligulacephalin B & L. stenocephala & root & {$[120]$} \\
\hline $4-52$ & (-)-Ligulacephalin B & L. stenocephala & root & [120] \\
\hline
\end{tabular}




\begin{tabular}{|c|c|c|c|c|}
\hline $4-53$ & $(+)$-Ligulacephalin $\mathrm{C}$ & L. stenocephala & root & [120] \\
\hline $4-54$ & $(-)$-Ligulacephalin C & L. stenocephala & root & [120] \\
\hline 4-55 & Stenocephalin B & L. stenocephala & root & [118] \\
\hline 4-56 & Stenocephalin C & L. stenocephala & root & [118] \\
\hline 4-57 & $(R)-(+)$-Ligulaodonin A & L. odontomanes & whole plant & [101] \\
\hline 4-58 & Ligustenin A & L. stenocephala & root & [119] \\
\hline 4-59 & $\begin{array}{l}(2 \alpha, 3 \beta, 5 \alpha)-2-(\text { Acetyloxy)-9-methoxy-5-(methoxycarbonyl)-2,3- } \\
\text { dimethylheptano-5-lactone }\end{array}$ & L. dentata & root & [83] \\
\hline 4-60 & $\begin{array}{l}(2 \beta, 4 \beta) \text {-2-Ethyl-5-hydroxy-5-(methoxy-carbonyl)-4,5-dimethylpentano-4- } \\
\text { lactone }\end{array}$ & L. dentata & root & [83] \\
\hline 4-61 & Euparin & L. caloxantha & root and rhizome & [121] \\
\hline 4-62 & 6-Methoxy-euparin & L. caloxantha & root and rhizome & [121] \\
\hline 4-63 & 3,4-Dicaffeoylquinic acid & $\begin{array}{l}\text { L. fischeri var. spiciformis and } \\
\text { L. stenocephala }\end{array}$ & $\begin{array}{l}\text { whole plant and } \\
\text { leaves }\end{array}$ & $\begin{array}{l}{[122]} \\
{[123]}\end{array}$ \\
\hline 4-64 & 3,5-Dicaffeoylquinic acid & L. stenocephala & leaves & [123] \\
\hline
\end{tabular}

used as a folk anti-insect agent.

\section{Biological Activities}

2.1 Antibacterial Activity: In 2003, Li et al reported that eremophilane sesquiterpenoid lactones 1-20 and 1-51, isolated from L. sagitta, showed antibacterial activity against Staphylococcus aureus, Bacillus subtilis and Escherichia coli according to the paper-disk method ${ }^{26}$. In 2009, another eremophilane sesquiterpenoid lactone 1-41, from $L$. hodgsonii, was reported showing weak antibacterial activity against Bacillus subtilis with the MIC of $128 \mu \mathrm{g} / \mathrm{mL}^{37}$.

2.2 Cytotoxic Activity: In 2006, Wu et al reported the cytotoxic activities of eremoligularin (1-130) and bieremoligularolide (1-268). The result revealed that bieremoligularolide (1-268) showed strong cytotoxicities: $I C_{50}$ $=5.5,16.1$, and $8.9 \mu \mathrm{M}$ against HL-60, SMMC-7721, and HeLa cells, respectively. However, eremoligularin (1-130) showed no cytotoxicity against the above three cells $\left(\mathrm{IC}_{50}>\right.$ $100 \mu \mathrm{M})^{15}$. In addition, in 2008, an eremophilane sesquiterpenoid 1-152, obtained from L. veitchiana, was reported to exhibited significant inhibiting activities on the growth of lung-cancer (A549) and stomachcancer (BCG823) cell lines, with $\mathrm{IC}_{50}$ values of 10.27 (A549) and 31.14 (BCG823) $\mu \mathrm{g} / \mathrm{mL}$, respectively ${ }^{67}$. While in 2010, an bisabolane sesquiterpene 1-241 was found showing significant cytotoxicity against human lung carcinoma (A-549), human breast adenocarcinoma (MCF-7), epidermoid carcinoma of the nasopharynx (KB), and vincristine-resistant nasopharyngeal (KBVIN) cell lines, with $\mathrm{EC}_{50}$ values of 3.4 (A549), 0.8 (MCF-7), $1.0(\mathrm{~KB})$, and 0.9 (KBVIN) $\mu \mathrm{g} / \mathrm{mL}$, respectively ${ }^{79}$.

2.3 Protein Tyrosine Phosphatase Inhibitory Activity: In 2009, an eremophilane lactone 1-43, from the roots of $L$. fischeri, was evaluated for the inhibitory activity against protein tyrosine phosphatase (PTP1B) in vivo by Deng et al ${ }^{23}$. The experiment data indicated moderate inhibitory activity with $\mathrm{IC}_{50}=1.34 \mu \mathrm{M}$.

2.4 Insecticidal and Antifeedant Activities: The plant $L$. caloxantha has been used as a folk medicine in the Naxi nationality in Yunnan province for years. In 2005, a phytochemical investigeation on the roots and rhizomes of this plant by $\mathrm{Li}$ et al led to the isolation of two benzofuran compounds, euparin (4-61) and 6-methoxy-euparin (4-62) ${ }^{121}$. The bioactivity assay revealed that both of the two compounds showed significant insecticidal and antifeedant activities. This conclusion may provide an explanation why $L$. caloxantha is
2.5 Antihepatotoxicity and Antioxidative Activity: It has been reported that the $\mathrm{MeOH}$ extract (LFS) of L. fischeri var. spiciformis and its active component, 3,4-dicaffeoylquinic acid (DCQA) (4-63), showed significant antihepatotoxicity, the action mechanism of which was investigated by Choi et al in $2004^{122}$. The result showed that both LFS and DCQA resultantly prevented hepatotoxicity via antioxidative mechanism. Thus, it was proposed that antihepatotoxicity of LFS was based on the antioxidative action of DCQA.

2.6 Antithrombotic and Anticoagulating Activity: In 2008, Yoon et al reported that the leaf extract of $L$. stenocephala showed the highest anti-platelet aggregating activity. The active fraction inhibited the platelet aggregation up to above $80 \%$ and its blood coagulating time also showed similar effect to aspirin $(0.2 \mu \mathrm{g} / \mathrm{mL})$, known as an antithrombus compound. An activity-guided seperation resulted in two antithrombus active compounds as 3,4-dicaffeoylquinic acid (4-63) and 3,5-dicaffeoylquinic acid (4-64). A further assay showed that the two active compounds has not only antiplatelet aggregating activity, but also has anticoagulating activity $^{123}$.

\section{Conclusions}

This review summarized the secondary metabolites reported from Ligularia species as well as their biological activities in recent decades. These conclusions indicate that Ligularia species may be a rich source of natural products with chemical and biological diversity.

\section{Acknowledgments}

This work was supported by the Important Directional Project of the Chinese Academy of Sciences (No. KSCX2-EW-R15) and the National Natural Science Foundation of China (No. 21075127).

Open Access This article is distributed under the terms of the Creative Commons Attribution License which permits any use, distribution, and reproduction in any medium, provided the original author(s) and source are credited.

\section{References}

[1] Liu, X.; Wu, Q. X.; Wei, X. N.; Shi, Y. P. Helv. Chim. Acta 2007, 90, 1802-1810.

[2] Li, P. L.; Zhang, Z. X.; Jia, Z. J. Chem. Lett. 2008, 37, 308-309.

\section{望 Springer}


[3] Xie, W. D.; Weng, C. W.; Li, X.; Row, K. H. Helv. Chim. Acta 2010, 93, 1983-1989.

[4] Liu, Q.; Shen, L.; Wang, T. T.; Chen, C. J.; Qi, W. Y.; Gao, K. Food Chem. 2010, 122, 55-59.

[5] Fei, D. Q.; Li, S. G.; Liu, C. M.; Wu, G.; Gao, K. J. Nat.l Prod. 2007, 70, 241-245.

[6] Wang, C. F.; Zhao, Y.; Shi, S. Y.; Li, J. P.; Zhang, Z. Z.; Liu, Y. Z. Chem. Nat. Comp. 2010, 46, 184-186.

[7] Wu, Q. X.; Shi, Y. P,; Jia, Z. J. Nat.Prod. Rep. 2006, 63, 699-734.

[8] Yang, J. L.; Liu, L. L.; Shi, Y. P. Tetrahedron Lett. 2009, 50, 6315-6317.

[9] Wu, Q. X.; Shi, Y. P.; Yang, L. Org. Lett. 2004, 6, 2313-2316.

[10] Yang, J. L.; Liu, L. L.; Shi, Y. P. Planta Med. 2011, 77, 271-276.

[11] Yang, J. L.; Wang, R.; Liu, L. L.; Shi, Y. P. Planta Med. 2011, 77, 362-367.

[12] Yang, J. L.; Liu, L. L.; Wang, B. G.; Shi, Y. P. Biochem. Syst. Ecol. 2010, 38, 850-852.

[13] Liu, J. X.; Wei, X. N.; Shi, Y. P.; Lu, R. H. Chin. Chem. Lett. 2005, 16, 1618-1620.

[14] Zhang, W. J.; Li, X. H.; Shi, Y. P. J. Nat. Prod. 2010, 73, 143146.

[15] Wu, Q. H.; Liu, C. M.; Chen, Y. J.; Gao, K. Helv. Chim. Acta 2006, 89, 915-922.

[16] Xu, J. Q.; Hu, L. H. Helv. Chim. Acta 2009, 92, 357-361.

[17] Zhang, Z. X.; Fei, D. Q.; Jia, Z. J. Helv. Chim. Acta, 2008, 91, $1045-1052$.

[18] Jia, Z. J.; Zhao, Y.; Tan, R. X. J. Nat. Prod. 1993, 56, 494-499.

[19] Li, L.; Xu, L. W.; Jiang, Y. F.; Xi, C. J.; Wang, H. Q.; Suo, Y. R. Z. Naturforsch. 2004, 59b, 921-924.

[20] Wang, W. S.; Dai, X.; Hong, L. Y.; Lu, P.; Feng, J. C.; Jiao, Y. G. Helv. Chim. Acta 2008, 91, 1118-1123.

[21] Zhao, Y.; Jia, Z. J.; Peng, H. R. J. Nat. Prod. 1995, 58, 13581364

[22] Li, L.; Xu, L. W.; Wang, H. Q. Helv. Chim. Acta 2004, 87, $1125-1129$.

[23] Deng, M. C.; Dong, W. W.; Jiao, W.; Lu, R. H. Helv. Chim. Acta 2009, 92, 495-501

[24] Tori, M.; Watanabe, A.; Matsuo, S.; Okamoto, Y.; Tachikawa, K.; Takaoka, S.; Gong, X.; Kuroda, C.; Hanai, R. Tetrahedron, 2008, 64, 4486-4495.

[25] Park, H. J.; Kwon, S. H.; Yoo, K. O.; Sohn, I. C.; Lee, K. T.; Lee, H. K. Planta Med. 2000, 66,783-784.

[26] Li, X. Q.; Gao, K.; Jia, Z. J. Planta Med. 2003, 69, 356-360.

[27] Jia, Z. J.; Zhao, Y.; Tan, R. X.; Yang, L. Phytochemistry 1992, 31, 199-201.

[28] Zhao, Y.; Jia, Z. J. Chin. Chem. Lett. 1993, 4, 323-326.

[29] Jia, Z. J.; Zhao, Y.; Tan, R. X. Planta Med. 1992, 58, 365-367.

[30] Liu, J. X.; Wei, X. N.; Shi, Y. P. Planta Med. 2006, 72, 175-179.

[31] Wu, Q. X.; Yang, A. M.; Shi, Y. P. Tetrahedron 2005, 61, 10529-10535.

[32] Nakatani, M.; Maeda, S.; Higashi, K.; Kurawaki, J.; Okamura, H.; Iwagawa, T. Heterocycles 2003, 60, 373-378.

[33] Zhao, Y.; Jia, Z. J.; Tan, R. X.; Yang, L. Phytochemistry 1992, 31, 2785-2787.

[34] Tori, M.; Okamoto, Y.; Tachikawa, K.; Mihara, K.; Watanabe, A.; Sakaoku, M.; Takaoka, S.; Tanaka, M.; Gong, X.; Kuroda, C.; Hattori, M.; Hanai, R. Tetrahedron 2008, 64, 9136-9142.

[35] Zhang, Z. X.; Lin, C. J.; Li, P. L.; Jia, Z. J. Planta Med. 2007, 73, $585-590$.

[36] Li, W. X.; Lei, M.; Fei, D. Q.; Gao, K. Planta Med. 2009, 75, 635-640.

[37] Xu, Y. J.; Nan, Z. D.; Li, W. H.; Huang, H. L.; Yuan, C. S. Helv. Chim. Acta 2009, 92, 209-216.

[38] Liu, J. Q.; Zhang, M.; Zhang, C. F.; Qi, H. Y.; Bashall, A.; Bligh, S. W. A.; Wang, Z. T. Phytochemistry 2008, 69, 2231-2236.

[39] Li, E. W.; Gao, K.; Jia, Z. J. Chin. Chem. Lett. 2004, 15, 194 196.

[40] Li, P. L.; Jia, J. Z. Helv. Chim. Acta 2008, 91, 1717-1727.

[41] Xu, J. Q.; Hu, L. H. Helv. Chim. Acta 2008, 91, 951-957.
[42] Tori, M.; Honda, K.; Nakamizo, H.; Okamoto, Y.; Sakaoku, M.; Takaoka, S.; Gong, X.; Shen, Y. M.; Kuroda, C.; Hanai, R. Tetrahedron 2006, 62, 4988-4995.

[43] Wu, Q. X.; Shi, Y. P.; Yang, L. Chin. Chem. Lett. 2004, 15, 1441-1444.

[44] Chen, H. M.; Jia, Z. J. Chin. Chem. Lett. 1991, 2, 849-852.

[45] Wu, Q. X.; Shi, Y. P.; Yang, L. Planta Med. 2004, 70, 479-482.

[46] Tori, M.; Nakamizo, H.; Mihara, K.; Sato, M.; Okamoto, Y.; Nakashima, K.; Tanaka, M.; Saito, Y.; Sono, M.; Gong, X.; Shen, Y. M.; Hanai, R.; Kuroda, C. Phytochemistry, 2008, 69, 1158-1165

[47] Han, Y. F.; Pan, J.; Gao, K.; Jia, Z. J. Chem. Pharm. Bull. 2005, 53, 1338-1341.

[48] Li, Y. S.; Wang, Z. T.; Zhang, M.; Zhou, H.; Chen, J. J.; Luo, S. D. Planta Med. 2004, 70, 239-243.

[49] Chen, H. M.; Cai, M. S.; Jia, Z. J. Phytochemistry 1997, 45, 1441-1444.

[50] Chen, H. M.; Zhang, K. Q.; Cai, M. S.; Jia, Z. J. Indian J. Chem. 1998, 37b, 720-722.

[51] Shi, S. Y.; Wu, D. Y.; Gong, X.; Peng, H.; Zhang, R. P.; Zhou, X.; Hao, X. J.; Jia, Z. J.; StÖckigt, J.; Zhao, Y. Chin. Chem. Lett. 2007, 18, 59-61.

[52] Nagano, H.; Iwazaki, Y.; Matsushima, M.; Sato, M.; Gong, X.; Shen, Y. M.; Hirota, H.; Kuroda, C.; Hanai, R. Chem. Biodiv. 2007, 4, 2874-2888.

[53] Fu, B.; Zhu, Q. X.; Yang, X. P.; Jia, Z. J. Pharmazie 2002, 57, 275-278.

[54] Zhang, W. J.; Qi, H. Y.; Shi, Y. P. Planta Med. 2010, 76, 159 164.

[55] Jia, Z. J.; Zhao, Y. J. Nat. Prod. 1994, 57, 146-150.

[56] Zhang, M.; Zhang, Z. F.; Wang, Z. T. Acta Pharm. Sin. 2005, 40 $529-532$.

[57] Jun, Z.; Wu, H.; Huang, K. X.; Shi, S. Y.; Peng, H.; Sun, X. F.; Chen, L. R.; Zhang, Q. X.; Zhang, Q. J.; Hao, X. J.; StÖckigt, J.; Li, X. K.; Zhao, Y.; Qu, J. Chin. Chem. Lett. 2008, 19, 13191322

[58] Wang, Q.; Mu, Q.; Shibano, M.; Morris-Natschke, S. L.; Lee, K. H.; Chen, D. F. J. Nat. Prod. 2007, 70, 1259-1262.

[59] Tori, M.; Tanio, Y.; Okamoto, Y.; Saito, Y.; Gong, X.; Kuroda, C.; Hanai, R. Heterocycles 2008, 75, 2029-2034.

[60] Nagano, H.; Matsushima, M.; Yamada, H.; Hanai, R.; Gong, X.; Kuroda, C. Nat. Prod. Comm. 2010, 5, 1-4.

[61] Nagano, H.; Kanda, M.; Yamada, H.; Hanai, R.; Gong, X.; Kuroda, C. Helv. Chim. Acta 2010, 93, 1945-1952.

[62] Chen, H. M.; Jia, Z. J.; Yang, L. Phytochemistry 1992, 31, 2146 2147

[63] Wu, Q. H.; Wang, C. M.; Cheng, S. G.; Gao, K. Tetrahedron Lett. 2004, 45, 8855-8858.

[64] Wang, W. S.; Zhu, Q. X.; Gao, K.; Jia, Z. J. J. Chin. Chem. Soc. 2000, 47, 1291-1293.

[65] Tan, A. M.; He, H. P.; Yang, H.; Zhang, M.; Wang, Z. T.; Hao, X. J. Acta Pharm. Sin. 2003, 38, 924-926.

[66] Li, Y.; Shi, Y. P. Hel. Chim. Acta 2006, 89, 870-875.

[67] Wang, C. F.; Zhao,Y.; Liu, Y. Z.; Zhang, Z. Z. Helv. Chim. Acta 2008, 91, 1712-1716.

[68] Zhao, Y.; Peng, H. R.; Jia, Z. J. J. Nat. Prod. 1994, 57, 16261630

[69] Yang, L.; Peng, H. R.; Jia, Z. J. Chin. Chem. Lett. 1995, 6, 875876.

[70] Li, Y. S.; Wang, Z. T.; Zhang, M.; Chen, J. J.; Luo, S. D. Nat. Prod. Res. 2004, 18, 99-104.

[71] Gao, K.; Jia, Z. J. Phytochemistry 1998, 49, 167-169.

[72] Baba, H.; Yaoita, Y.; Kikuchi, M. J. Tohoku Pharm. Univ. 2007, 54, 53-56.

[73] Gao, K.; Jia, Z. J. J. Lanzhou Univ. 2000, 36, 127-128.

[74] Guo, Z.; Weng, C. W.; Liu, W. X.; Shen, T. J. Chem. Res. 2010 390-391.

[75] Yoshinori, S.; Masato, H.; Yuko, I.; Yuriko, T.; Kanako, M.; 
Yoko, S.; Miho, F.; Misato, S.; Anna, S.; Xun, C.; Chiaki, K.; Xun, G.; Ryo, H.; Motoo, T. Tetrahedron 2011, 67, 2220-2231.

[76] Naya, K.; Okayama, T.; Fujiwara, M.; Nakata, M.; Ohtsuka, T.; Kurio, S. Bull. Chem. Soc. Japn. 1990, 63, 2239-2245.

[77] Li, P. L.; Wang, C. M.; Zhang, Z. X.; Jia, Z. J. Tetrahedron 2007, 63, 12665-12670.

[78] Zhao, Y.; Parsons, S.; Smart, B. A.; Tan, R. X.; Jia, Z. J.; Sun, H. D.; Rankin, D. W. H. Tetrahedron 1997, 53, 6195-6208

[79] Wang, Q.; Chen, T. H.; Bastow, K. F.; Lee, K. H.; Chen, D. F. J. Nat. Prod. 2010, 73, 139-142.

[80] Liu, C. M.; Wang, H. X.; Wei, S. L.; Gao, K. Helv. Chim. Acta 2008, 91, 308-316.

[81] Onuki, H.; Yamazaki, M.; Nakamura, A.; Hanai, R.; Kuroda, C.; Gong, X.; Shen, Y. M.; Hirota, H. J. Nat. Prod. 2008, 71, 520524.

[82] Liu, C. M.; Fei, D. Q.; Wu, Q. H.; Gao, K. J. Nat. Prod. 2006, 69, 695-699.

[83] Baba, H.; Yaoita, Y.; Kikuchi, M. Helv. Chim. Acta 2007, 90, $1028-1037$.

[84] Tan, A. M.; He, H. P.; Zhang, M.; Wang, Z. T.; Hao, X. J. Helv. Chim. Acta 2007, 90, 101-104.

[85] Gao, K.; Yang, L.; Jia, Z. J. Indian J. Chem. 1997, 36b, 715-718.

[86] Baba, H.; Yaoita, Y.; Kikuchi, M. Helv. Chim. Acta 2007, 90, $1302-1312$.

[87] Gao, X.; Lin, C. J.; Xie, W. D.; Shen, T.; Jia, Z. J. Helv. Chim. Acta 2006, 89, 1387-1394

[88] Gao, X.; Xie, W. D.; Jia, Z. J. J. Asian Nat. Prod. Res. 2008, $10,185-192$.

[89] Wang, Q.; Chen, D. F. Helv. Chim. Acta 2007, 90, 2432-2437.

[90] Gao, K.; Jia, Z. J. Chem. Res. Chin. Univ. 1999, 15, 70-72.

[91] Jia, Z. J.; Chen, H. M. Phytochemistry 1991, 30, 3132-3134

[92] Wu, Q. X.; Wei, Q. Y.; Shi, Y. P. Pharmazie 2006, 61, 241-243.

[93] Marco, J. A.; Sanz, J. F.; Yuste, A.; Rustaiyan, A. Liebigs Ann. Chem. 1991, No. 9, 929-931.

[94] Wang, B. G.; Yang, L.; Jia, Z. J.; Chen, H. M. Indian J. Chem. 1998, 37b, 669-671.

[95] Li, Y. S.; Li, S. S.; Wang, Z. T.; Luo, S. D.; Zhu, D. Y. Nat. Prod. Res., Part A 2006, 20, 1241-1245.

[96] Zhang, Z. X.; Fei, D. Q.; Jia, Z. J. Bull. Chem. Soc. Jpn. 2008, $81,1007-1011$.

[97] Sun, X. B.; Xu, Y. J.; Qiu, D. F.; Yuan, C. S. Helv. Chim. Acta 2007, 90, 1705-1711.

[98] Huang, H. L.; Xu, Y. J.; Liu, H. L.; Liu, X. Q.; Shang, J. N.; Han, G. T.; Yao, M. J.; Yuan, C. S. Phytochemistry 2011, 72, 514517.

[99] Fei, D. Q.; Wu, Q. H.; Li S. G.; Gao, K. Chem. Pharm. Bull. 2010, $58,467-469$.
[100] Lee, K. T.; Koo, S. J.; Jung, S. H.; Choi, J.; Jung, H. J.; Park, H. J. Arch. Pharm. Res. 2002, 25, 820-823.

[101] Fei, D. Q.; Wu, G.; Liu, C. M.; Gao, K. Chem. Pharm. Bull. 2007, 55, 577-579.

[102] Zhu, H.; Tu, P. F. Z. Naturforsch. 2004, 59b, 1063-1066.

[103] Zhao, Y.; Tian, J.; Jia, Z. J.; Sun, H. D. Acta Bot. Yunnan. 1995, $17,356-358$.

[104] Ma, B.; Shi, Y. P.; Jia, Z. J. Planta Med. 1997, 63, 573-574.

[105] Toyoda, K.; Yaoita, Y.; Kikuchi, M. J. Tohoku Pharm. Univ. 2006, 53, 51-55.

[106] Tan, A. M.; Wang, Z. T.; He, H. P.; Zhang, M.; Hao, X. J. Heterocycles 2003, 60, 1195-1198.

[107] Lin, G.; Rose, P.; Chatson, K. B.; Hawes, E. M.; Zhao, X. G.; Wang, Z. T. J. Nat. Prod. 2000, 63, 857-860.

[108] Tan, A. M.; Li, Y. S.; Yang, H.; Wang, Z. T.; He, H. P.; Zhang, M.; Hao, X. J. Chin. Chem. Lett. 2004, 15, 68-70.

[109] Zhang, C. F.; Wang, Q.; Zhang, M. J. Asian Nat.Prod.Res. 2009, 11, 339-344.

[110] Li, Y. S.; Wang, Z. T.; Zhang, M.; Luo, S. D.; Chen, J. J. Nat. Prod. Res. 2005, 19, 125-129.

[111] Gao, X.; Jia, Z. J. Chin. Chem. Lett. 2008, 19, 71-72.

[112] Ma, B.; Gao, K.; Shi, Y. P.; Jia, Z. J. Phytochemistry 1997, 46, 915-919.

[113] Yan, F. L.; Wang, A. X.; Jia, Z. J. J. Chem. Res. 2004, 11, 742743.

[114] Zhang, M.; Wang, Z. T.; Qin, H. L.; Zhao, X. G.; Xu, G. J.; Li, J. X.; Namba, T. Chin. Chem. Lett. 2002, 13, 620-622.

[115] Shi, S. Y.; Hu, M. H.; Wu, D. Y.; Zhou, C. X.; Mo, J. X.; Xu, J. H.; Chen, L. R.; Dou, H.; Peng, H.; Hao, X. J.; Stockigt. J.; Zhao, Y. Nat. Prod. Res., Part A 2008, 22, 628-632.

[116] Gao, K.; Wang, W. S.; Jia, Z. J. Phytochemistry 1998, 47, 269272.

[117] Zhao, Y.; Jia, Z. J.; Yang, L.; Wang, J. G. Chin. Chem. Lett. 1993, 4, 895-898.

[118] Yan, F. L.; Wang, A. X.; Jia, Z. J. Pharmazie 2005, 60, 155159.

[119] Yan, F. L.; Wang, A. X.; Jia, Z. J. J. Chin. Chem. Soc. 2004, 51, $863-868$.

[120] Toyoda, K.; Yaoita, Y.; Kikuchi, M. Chem. Pharm. Bull. 2005, $53,1555-1558$

[121] Li, Y. S.; Wang, Z. T.; Zhang, M.; Tan, A. M.; Chen, L. Chin. Tradit. Herb. Drugs 2005, 36, 335-337.

[122] Choi, J.; Park, J. K.; Lee, K. T.; Park, K. K.; Kim, W. B.; Lee, J. H.; Jung, H. J.; Park, H. J. Nat. Prod. Sci. 2004, 10, 182-189.

[123] Yoon, M. H.; Cho, CW.; Lee, J. W.; Kim, Y. S.; An, G. H.; Lim, C. H. Nat. Prod. Sci. 2008, 14, 62-67. 